\title{
Collicular Microstimulation During Passive Rotation Does Not Generate Fixed Gaze Shifts
}

\author{
A. D. VAN BEUZEKOM AND J.A.M. VAN GISBERGEN \\ Department of Biophysics, University of Nijmegen, 6500 HB Nijmegen, The Netherlands
}

Received 13 August 2001; accepted in final form 30 January 2002

Van Beuzekom, A. D. and J.A.M. Van Gisbergen. Collicular microstimulation during passive rotation does not generate fixed gaze shifts. J Neurophysiol 87: 2946-2963, 2002; 10.1152/jn.00675.2001. We investigated whether saccades evoked by electrical stimulation (E-saccades) in the superior colliculus can compensate for passive sinusoidal head rotation in yaw so as to keep the rapid gaze shift constant. After accounting for variations in E-saccade onset position, we found significant horizontal metric changes, proportional to head velocity, in 31 of 37 experiments in 2 monkeys. Vertical effects were small. In a substantial fraction of the experiments (14/37), these metric changes represented significant but often insufficient compensatory adjustments in the horizontal component, opposite to the direction of head movement. However, very robust violations of gaze-shift constancy were remarkably common: significant anticompensatory changes in the horizontal component occurred in 17/37 experiments. In these cases, typically involving larger E-saccades, the horizontal component increased in size with rotation into the half field containing the E-saccade and became smaller during opposite rotation. Further analysis showed that, instead of showing a dichotomy, the metric effect actually varied along a continuum from compensatory to strongly anticompensatory. In addition to these metric changes, we found a robust kinematic effect of head rotation in metrically matched E-saccades. In all experiments where the effect was significant $(34 / 37)$, horizontal peak velocity increased for rotation into the half field where the E-saccade was directed and decreased for opposite rotation. This kinematic effect was again proportional to head velocity and predominant in the horizontal component. Comparison of yaw and pitch rotation at the same stimulation site showed that both expressions of vestibular-saccade interaction (metric and kinematic) tended to align with the direction of rotation. The component-specific nature of the modulation suggests that the effects may have been caused by convergence of saccadic and vestibular signals at a component-coding stage downstream of the colliculus. We suggest that the quick-phase system got access to the common pulse generator as soon as the collicular stimulation had opened the pause-cell gate. Adding such an anticompensatory signal would act to increase the E-saccade horizontal component when the monkey was rotated in the same direction and bring about a decrease in size and peak velocity when it was opposite. In the large majority of experiments the metric changes failed to maintain gaze-shift constancy, either because they were in the wrong direction or because they were too small. Possible reasons for this major departure from the properties of natural gaze shifts are discussed.

\section{N T R O D U C T I O N}

There is general agreement that the control of rapid eye movements relies on a common circuit involving burst neurons

Address for reprint requests: A. D. Van Beuzekom, 231 Dept. of Biophysics, University of Nijmegen, PO Box 9101, 6500 HB Nijmegen, The Netherlands. that is called into action both for goal-directed saccades to a selected target and during quick phases generated by the vestibuloocular reflex (VOR). Studies on VOR quick phases have typically side-stepped the issue how they might interact with goal-directed saccades. However, preliminary findings by Kitama et al. (1992) in the cat may have interesting implications for this question. These authors reported saccade-vestibular interactions in electrically induced collicular saccades (E-saccades) involving an anticompensatory vestibular signal. Since, if confirmed, such results would seem to provide an interesting window on the neglected topic of saccade-quick phase interaction, we have performed similar experiments in the passively rotated monkey. Our major motivation to pursue the basic observation of Kitama and co-workers is that it argues against the notion of gaze constancy that has become a key feature of most current gaze-control models. To provide a more detailed account of the relevant issues, the subsequent sections will briefly review the literature on the neural control of fast eye movements and on current ideas concerning saccade-vestibular interaction.

\section{Neural control of rapid eye movements}

It is well-established that signals for the generation of goaldirected saccades and quick phases of nystagmus finally converge on a common brain stem circuit, involving excitatory burst cells (EBNs) and omnidirectional pause neurons (Fig. 1), known as the pulse generator (for reviews see Hepp et al. 1989; Keller 1991; Moschovakis et al. 1996; Scudder et al. 2002). EBNs specialized for horizontal and for vertical/torsional rapid eye movements have been identified in the pontine reticular formation and the rostral midbrain, respectively. They burst both during goal-directed saccades and quick phases into their ON-direction.

Omnipause neurons show a steady discharge during fixation and slow phases of nystagmus, but cease firing during both types of rapid eye movements in any direction. By disinhibiting burst neurons in this fashion, the omnipause neurons gate the pulse generator and control the timing of rapid eye movements (Gandhi and Keller 1999; Keller 1974).

In principle, this picture of how the pulse-generator circuit works can explain the stereotyped and intermittent nature of rapid eye movements (Robinson 1975; Van Gisbergen et al. 1981; Zee et al. 1976). The wider question of how and when

The costs of publication of this article were defrayed in part by the payment of page charges. The article must therefore be hereby marked "advertisement" in accordance with 18 U.S.C. Section 1734 solely to indicate this fact. 


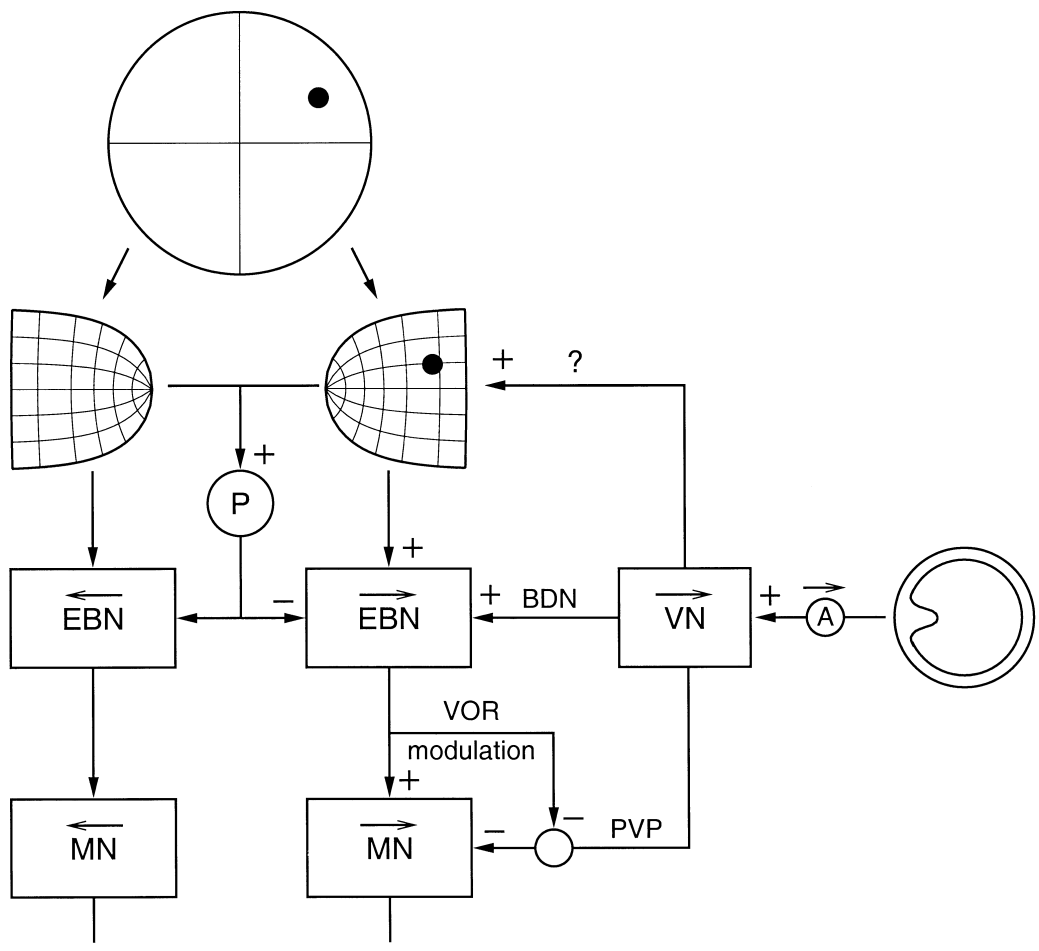

FIG. 1. Control signals underlying the generation of goaldirected rapid eye movements and vestibular quick phases. The scheme illustrates the final common pathway for goal-directed saccades and vestibular quick phases. To generate a saccade to a visual target (top), the superior colliculus (2nd row) sends a displacement signal to the burst cells (EBNs) downstream. When the bursters are enabled by the gate, embodied by the omnipause neurons $(\mathrm{P})$, they send an eye-velocity signal to the motoneurons (MN). To generate quick phases during head rotation, the head velocity signal from the semicircular canals (far right) is sent by primary afferents (A) to the vestibular nucleus (VN). After further processing of this signal, burster driving neurons (BDNs) activate the EBNs. An alternative and more hypothetical pathway for generating quick phases, through the colliculus is also indicated (?). In parallel, the position-vestibular-pause cells (PVP) carry a compensatory vestibuloocular reflex (VOR) signal, that is suppressed during large gaze shifts. For simplicity, all neurons contributing to rightward rapid eye movements have been depicted in the right half of the scheme. Internal feedback signals have been omitted.

this system is called into action has been pursued mainly in studies concentrating on more central saccadic control mechanisms (for reviews, see Guitton 1991; Sparks and HartwichYoung 1989; Wurtz 1996). This work has provided clear evidence that the pathways for saccades to visual, auditory, and tactile targets have already converged at the level of the superior colliculus (SC), which plays an important role in the sensory-motor transformation for the control of saccadic eye movements (Groh and Sparks 1996; Jay and Sparks 1987; Sparks 1986). Burst cells in the deeper layers of the SC exhibit a vigorous burst, tightly linked to saccade onset. In contrast to the temporal coding of saccade components in EBNs, collicular neurons are organized into a two-dimensional topographic map, representing the contralateral hemifield, that specifies the relation between the locus of activity in the map and the saccade vector (Robinson 1972) (see also Fig. 1). Collicular saccade-related burst cells have limited movement fields (Schiller and Stryker 1972; Wurtz and Goldberg 1972).

The generation of quick phases by vestibular signals has received much less attention. Experiments in the cat (Kitama et al. 1995; Ohki et al. 1988; see Markham 1996 for review) have suggested an important role for burster driving neurons (BDNs) in activating the pulse generator during these rapid eye movements (see Fig. 1). The possible role of the SC in the control of quick phases has received only very scant attention, but there is some evidence that quick phases also have a neural representation in the colliculus map. For example, Schiller and Stryker (1972) found that collicular burst cells that become active during goal-directed saccades, may also show movement-related activity during quick phases. Furthermore, Wurtz and Goldberg (1972) described cells in the SC that were active both before horizontal visually guided saccades and before quick phases of caloric nystagmus of equal amplitude. A systematic movement-field study, however, has never been undertaken so that virtually nothing is known on how the spatial distribution of this activity relates to the layout of the collicular map. Reversible-inactivation experiments in the monkey by Hepp et al. (1993) showed that the SC plays an essential role in the generation of voluntary and goal-directed saccades: after inactivation, hardly any saccades were made. Quick phases, on the other hand, could still be generated, although their peak velocities were clearly reduced.

\section{Earlier studies on saccade-vestibular interactions}

These results on the afferent signals to the pulse generator for saccades and quick phases, pictorially summarized in Fig. 1 , were mostly obtained in dedicated studies concentrating on either system that left open how they operate in conjunction. For example, the fact that gaze shifts often involve a combined eye-head movement immediately raises questions on how collicular targeting signals and oculomotor signals of vestibular origin are combined. The prevailing view is that the SC, long seen as an area for the control of eye saccades, is actually a control center for combined eye-head gaze shifts (Freedman et al. 1996; Freedman and Sparks 1997; Roucoux et al. 1980). If the brain decides that the head should contribute to a voluntary gaze shift, will this simply lead to the addition of the vestibularly driven eye movements that normally accompany head movements when there is no explicit target? Investigations concerning this question have mostly concentrated on the slowphase signal of the VOR and have provided mixed evidence for slow-phase suppression during large gaze shifts (Guitton and Volle 1987; Laurutis and Robinson 1986; Pélisson et al. 1988; Tabak et al. 1996; Tomlinson and Bahra 1986). Positionvestibular-pause (PVP) cells, which are thought to carry a VOR slow-phase signal, are inhibited during voluntary gaze shifts (Roy and Cullen 1998), possibly by inhibition mediated 
by the pulse generator (see Fig. 1). On this basis, it has been suggested that the PVPs may play a role in VOR suppression.

If gaze shifts can affect the generation of VOR slow phases, how about the quick-phase mechanism? While it is not hard to see a rationale for suppressing slow-phase signals, which would counteract the gaze shift, predicting the fate of the anticompensatory quick phases is not trivial from a theoretical point of view. In any case, since different neurons are involved, slow-phase suppression does not automatically imply quickphase suppression. Experimental and theoretical studies considering the issue of whether quick-phase signals may contribute to goal-directed rapid eye movements when the head is moving have been rare (but see, e.g., Barnes 1981; Barnes and Prosser 1981; Guitton and Volle 1987). Against this general background, the present study was undertaken with the objective to clarify how collicular saccadic commands and vestibularly related signals are combined when both systems are activated. We asked how E-saccade properties would be affected by yaw rotation in either direction, at various velocities. One potential scenario is that gaze-shift constancy is maintained by modifying the E-saccade to compensate for the ongoing head movement so as to keep the sum of eye and head movement constant (see Fig. 2). Alternatively, as suggested by earlier findings of Kitama et al. (1992) in the cat, vestibular stimulation may add an anticompensatory component to the E-saccade (see Fig. 2). Such loss of spatial constancy in anticompensatory E-saccades was seen in many experiments. The anticompensatory effect may come about when electrical SC stimulation opens the pause-cell gate, thereby allowing the expression of an anticompensatory movement from the quickphase system.

This result raised the important further question of whether this putative quick-phase contribution was generated at 1) the vectorial coding stage embodied by the SC motor map or 2) at downstream oculomotor centers carrying component-related signals. Since vector averaging (Robinson 1972) would be expected if quick phases have a collicular origin, the data were analyzed to check for this possibility. The results show no sign of averaging but rather reflect changes in the component

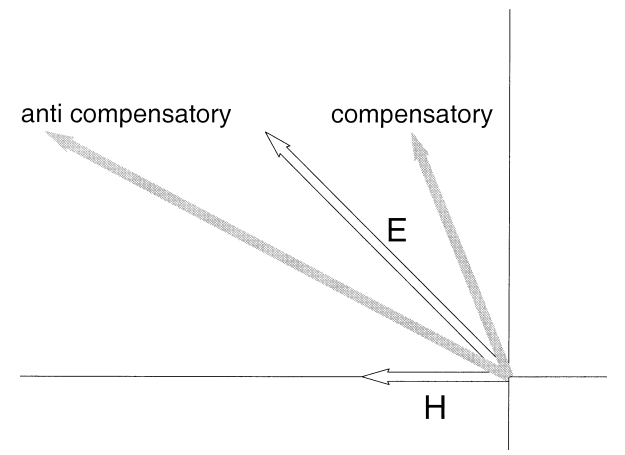

FIG. 2. Schematic illustration of possible effects of yaw rotation on Esaccades. To preserve gaze constancy during rotation, the saccade vector induced by superior colliculus (SC) stimulation in the stationary animal (E) must compensate for the head displacement occurring during the saccade $(\mathrm{H})$ such that the resulting E-vector equals E-H (gray vector labeled "compensatory"). According to the alternative scenario explained in the text, rotation may cause a manifest violation of gaze constancy by adding an anticompensatory signal to the E-saccade (gray vector labeled "anticompensatory"). aligned with the direction of rotation and therefore seem compatible with the second hypothesis.

\section{METHODS}

\section{Animal preparation and neurophysiological procedures}

The experiments were performed in two adult male rhesus monkeys (Macaca mulatta), weighing 6-7 kg, that had been trained to accurately fixate visual targets. The animals will be denoted as $B R$ and $G I$. All surgical and experimental procedures were reviewed and approved by the university committee for the use of experimental animals. Surgery was carried out in the local central animal facility, which was responsible for housing, feeding, and veterinary care.

SURGERY. To prepare the animals for chronical neurophysiological experiments, two separate sterile surgical procedures were performed under inhalant anesthesia with $\mathrm{N}_{2} \mathrm{O} / \mathrm{O}_{2}$ and ethrane, in combination with infusion of pentobarbital sodium. Blood pressure, heart rate, oxygen saturation, and body temperature were continuously monitored during surgery. The animal was artificially ventilated, and end-tidal $\mathrm{CO}_{2}$ was maintained around $4 \%$. A venous canule was inserted in a hind leg to allow a steady infusion of pentobarbital and saline. In the first surgical session, a thin gold-plated copper ring (diameter about $17 \mathrm{~mm}$ ) was implanted underneath the conjunctiva of the right eye, following a method similar to Judge et al. (1980). The ring, which became firmly attached to the eye by connective tissue, served to record two-dimensional eye movements (see below for details). In the second operation, a solid cap was tightly fitted to the skull. This was done by placing 14 tapered titanium bone screws (length $7.5 \mathrm{~mm}$, diameter $2.7 \mathrm{~mm}$ ) in drilled and tapped holes in the skull and embedding them in sterile orthopedic bone cement (Palacos). Four stainless steel bolts were fixed in the cement cap to allow rigid fixation of the head during experiments. A stainless steel recording chamber (11 mm ID) was stereotaxically implanted over a trephine hole, centered on the midline above the intersection of the midsagittal plane and the interaural line, such that both colliculi could be reached by microelectrode penetrations.

RECORDING OF NEURONAL ACTIVITY. The localization of the SC was based on a number of neurophysiological criteria (Melis and Van Gisbergen 1996). Extracellular activity in the SC was recorded using glass-coated tungsten microelectrodes (impedance 0.3-1.2 M $\Omega$ ). The electrode was placed inside a stainless steel guide tube to prevent damage to the tip during penetration of the dura and was moved downward by a hydraulic stepping motor (Trent Wells), mounted on the chamber. After amplification (BAK Electronics, Model A-1) and filtering (bandpass $100 \mathrm{~Hz}$ to $10 \mathrm{kHz}$ ), the electrode signal was monitored on an oscilloscope and fed into a level detector such that individual action potentials could be detected with a time resolution of $10 \mu \mathrm{s}$.

\section{Experimental procedures and setup}

All experiments were conducted in a completely dark room. While seated in a primate chair, the head-restrained monkey was rotated about either a vertical or horizontal axis through the cyclopean eye using a motor-driven vestibular stimulator. Chair position was measured using a digital position encoder with an angular resolution of $0.04^{\circ}$ (sample rate: $500 \mathrm{~Hz}$ ). Visual targets were presented using an array of red light-emitting diodes (LEDs). The array was attached to the vestibular stimulator, with the center LED on the monkey's naso-occipital axis at $0.39 \mathrm{~m}$ from the cyclopean eye, so that it moved with the monkey during rotations. LEDs were positioned on the intersections of seven circles at $5,10, \ldots, 35^{\circ}$ and 12 meridians every $30^{\circ}$. To calibrate the eye-ring signals, sessions started with a run in which the monkey made refixations from the central fixation LED to 
each of all 84 peripheral targets and maintained fixation as long as it was visible.

EYE POSITION RECORDING. Two-dimensional eye position relative to the head was recorded using the double-magnetic induction technique (Bour et al. 1984). Two oscillating perpendicular magnetic fields (horizontal: $48 \mathrm{kHz}$, vertical: $60 \mathrm{kHz}$ ) induced an eye-positiondependent electrical current in the implanted eye ring, which, in turn, induced secondary currents in a sensitive pickup coil that was mounted directly in front of that eye. A nulling coil, placed some distance away from the recording eye on a rigid manipulator, electronically canceled the primary eye-position-independent signal component induced by the magnetic fields. After amplification and demodulation by lock-in amplifiers (PAR 128A), the raw horizontal and vertical eye position signals were low-pass filtered $(-3 \mathrm{~dB}$ at $200 \mathrm{~Hz}$, 4th-order Bessel filter) and sampled with 12-bit resolution at $500 \mathrm{~Hz}$ per channel (CED 1401plus). This technique provides a high-resolution eye-position recording $\left(\sim 0.2^{\circ}\right.$ in all directions $)$ with only small nonlinearities that can be easily accounted for using a relatively simple calibration procedure (see Data analysis).

\section{Paradigms combining collicular microstimulation and vestibular stimulation}

VESTIBULAR STIMULATION. The experiments were designed to investigate how E-saccades were affected by vestibular stimulation compared with control data collected in absence of vestibular stimulation. In all sessions, vestibular stimulation was applied by rotation about the vertical axis, using a $0.15-\mathrm{Hz}$ sinusoidal profile with a maximal velocity of $66^{\circ} \%$ and an amplitude of $70^{\circ}$. In each run, the monkey was rotated continuously for $80 \mathrm{~s}$. In six sessions, the monkey subsequently was also rotated about a horizontal axis $\left(0.2 \mathrm{~Hz}, 57^{\circ} / \mathrm{s}\right.$, $45^{\circ}$ ). In these combined yaw-pitch sessions, we used the same velocity profile also for yaw rotation.

What was intended as yaw rotation led to slow phases with a small pitch component indicating that the monkey's sagittal head plane was not perfectly aligned with the earth-vertical rotation axis. The deviation was quantified by determining the relation between horizontal and vertical eye velocity. The slope of this relationship describes the deviation of the body axis from earth vertical. Deviations were small, $2.47 \pm 0.91^{\circ}$ (mean $\pm \mathrm{SD}$ ) for all sessions of monkey BR, $0.49 \pm$ $0.60^{\circ}$ for all experiments of monkey GI. Quick phases had a slight downward component in both rotation directions (see Fig. $3 C$ ), but this effect is probably related to drift compensation and would not be expected on the basis of the small pitch component in vestibular rotation.

ELECTRICAL STIMULATION. E-saccades were elicited by electrical stimulation of sites in the deeper layers of the caudal SC with a train of constant-current biphasic pulses (BAK Electronics, Model BPG-1). The train always had a pulse frequency of $500 \mathrm{~Hz}$ with each pulse lasting $0.2 \mathrm{~ms}$. We reliably elicited various amplitude saccades at 26 different collicular sites $\left(n_{B R}=20, n_{G I}=6\right)$. At each site, threshold was determined by gradually increasing the current strength. Stimulation threshold was defined as the current intensity where at least $90 \%$ of all stimulations led to a saccadic response while the monkey was scanning the experimental room. Experiments were then conducted with a current up to 2.5 times threshold. With respect to train duration, two different stimulation paradigms were in use. In the long paradigm $\left(n_{B R}=21, n_{G I}=5\right)$, we used train durations between 34 and $66 \mathrm{~ms}$, ensuring that the site-specific maximum amplitude E-saccade (Stanford et al. 1996) was elicited. At some sites $\left(n_{B R}=7, n_{G I}=\right.$ 4), we also applied a short 20-ms pulse train (short paradigm). Since the two paradigms typically yielded different E-saccades, we will describe the results as coming from different experiments. As a result, the total number of experiments (37) exceeds the number of sites.

To entice the monkey to look straight ahead, the central LED was flashed for $50 \mathrm{~ms}$ at random intervals between 3.3 and $4.7 \mathrm{~s}$, yielding
20 target presentations every $80 \mathrm{~s}$. We saw no consistent changes in the gain of the VOR at the time of electrical stimulation due to the presentation of the straight-ahead LED. The monkey was rewarded when moving gaze toward the remembered position of the flash. If after being rewarded the monkey's gaze was still near straight ahead (allowing maximally $10^{\circ}$ horizontal or vertical deviations), an electrical stimulation pulse train was applied (approximately 10 times every 80-s run). On average, there was a delay of roughly $750 \mathrm{~ms}$ between the flash and the electrical stimulation. Electrical stimulation occurred at various phases of the sinusoidal vestibular stimulation so that the effect of head velocity on E-saccades could be investigated. In a typical session, approximately 160 E-saccades were elicited during rotation, and about 190 during rest. In both paradigms, vestibular runs were alternated with control runs that were similar except for the absence of vestibular stimulation.

\section{Data analysis}

CALIBRATION OF EYE POSITION. Horizontal and vertical eye-coil signals were calibrated off-line using fixation data obtained in the eye-coil calibration run at the beginning of each experimental session. Two neural networks, one for each position component, were trained to fit the raw fixation data to the target locations, using a backpropagation algorithm based on the gradient-descent method of Levenberg-Marquardt (Matlab, the Mathworks). This algorithm was used to correct for the inherent nonlinearity of the double-magnetic induction technique. Each network consisted of two input units, representing the raw horizontal and vertical signal, three hidden units and one output unit, representing either the desired calibrated horizontal or vertical position signal (Melis and Van Gisbergen 1996). Raw eyecoil signals were subsequently calibrated by applying the resulting feed-forward networks. Calibration errors, i.e., the remaining error between actual target position and corrected signal, were typically less than $0.5^{\circ}$, on average. In all figures rightward and upward eye and chair position will be denoted as positive.

SACCADE DETECTION AND SELECTION. Saccade detection was performed on the calibrated eye position signals on the basis of separate velocity and acceleration/deceleration criteria for saccade onset and offset, respectively. All detection markings were checked by the experimenter and adjusted if necessary.

A rapid eye movement was considered an E-saccade if it started between 16 and $60 \mathrm{~ms}$ after the onset of the electrical stimulation train. E-saccades starting within $140 \mathrm{~ms}$ after a previous rapid eye movement were discarded from further analysis, to minimize temporal interaction effects of the type reported by several groups (Kustov and Robinson 1995; Nichols and Sparks 1995; Schlag et al. 1998). Metric and kinematic effects of rotation on E-saccades, analyzed by multiple linear regression (see RESULTS), did not show any relation with the intersaccadic interval.

\section{RE S U L T S}

To investigate the effect of vestibular stimulation, E-saccades were elicited by electrical microstimulation in the SC during passive head rotation and during rest. We will first provide data on the rapid and slow eye movements during vestibular stimulation and present an overview of the range of E-saccade vectors tested in the yaw-rotation experiments. Subsequently we describe the effect of vestibular stimulation on the metric and kinematic properties of E-saccades.

\section{Characteristics of nystagmic eye movements and range of tested E-saccade vectors}

Figure $3 A$ shows eye position traces during yaw rotation. At the time marked by the arrow, the electrical pulse train started 


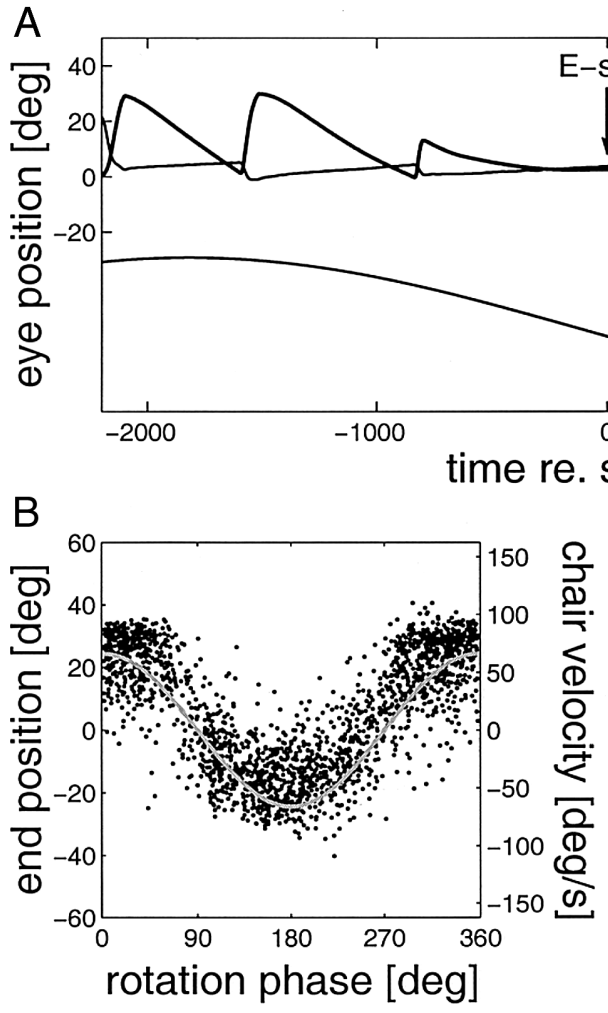

and after a short latency an E-saccade was made to the left and down. Since the E-saccade was elicited during ongoing sinusoidal rotation, it occurred against a background of nystagmus eye movements. The compensatory slow phase moved the eye in a direction opposite to the head rotation (average gain: $0.79 \pm 0.08$ ), while the anticompensatory quick phases prevented the eyes from getting stuck at the border of the oculomotor range. As noted before, the quick phases did not just reset the eye to the straight-ahead position, but typically ended at a more eccentric position, displaced into the direction of rotation (see, e.g., Chun and Robinson 1978). This so-called shift of the beating field is further illustrated in Fig. 3B, which shows quick phase end positions relative to the head as a function of head-velocity phase, revealing a clear relation between the end points of quick phases and instantaneous head velocity (gray line).

As shown by the gray-shaded density plot in Fig. $3 C$, the metric properties of quick phases in the absence of electrical stimulation showed wide scatter. The mean quick-phase vector $(+)$ had a horizontal component of $23.0^{\circ}$. The downward component of the mean quick-phase vector may reflect compensation for the clear upward eye position drift in the dark (see trace $V$ in $A$ ), as proposed by Fuchs et al. (1996). The upward drift (velocity $\sim 5^{\circ} / \mathrm{s}$ ) was present in both monkeys. For comparison, the open circles in Fig. $3 C$ represent all mean E-saccade vectors that were obtained in the control experiments performed in monkey $B R$ (stimulation in right SC). As can be seen, their amplitudes varied between 3.2 and $27.6^{\circ}$, and their directions covered the range from 113 to $243^{\circ}$.

\section{Dependence of E-saccade metrics on head velocity}

Qualitative observations by Kitama et al. (1992) in the cat suggest that vestibular stimulation may change E-saccade met-
FIG. 3. Properties of quick phases and E-sac을 cade vectors. $A$ : horizontal $(\mathrm{H})$ and vertical $(\mathrm{V})$ ㄹ. eye-position traces of nystagmus and an E-sac@- cade elicited during a half cycle of vestibular $\leftarrow \quad$ stimulation (chair). $B$ : the horizontal offset po$\frac{1}{D}$ sition of a large number of quick phases $(n=$ $2,000)$ as a function of head rotation phase to illustrate the shift of the beating field. Since the monkey's attention was attracted by flashing the central light-emitting diode (LED) every $4 \mathrm{~s}$ (see METHODS), all eye movements made until $750 \mathrm{~ms}$ after this flash were discarded in order to isolate pure vestibular quick phases. Quick phases did not reset the eye to the straight ahead position, but moved the eye to a more eccentric position. Note that there is a clear relation with instantaneous head velocity (gray line), with a small phase lead $\left(6.7^{\circ}\right)$. Quick phase end position was roughly linear with chair velocity (slope $0.47 \mathrm{~s}$ ). The distribution of quick-phase displacement vectors is presented in $C$, coded by gray levels. Quick-phase vectors $(n>29,000)$ ended up in 2 similar distributions, associated with leftward and rightward rotation. Note that the mean leftward and rightward quick-phase vectors (+) showed a clear downward vertical component of almost $5^{\circ}$, probably to compensate for upward eye position drift (see $A$ ). This downward bias was also observed in monkey GI (3.4 ${ }^{\circ}$, data not shown). The open circles represent the E-saccade vector end points that were elicited in monkey $B R$ by microstimulation in the right SC.

rics. We checked to what extent this is also true in the monkey and pursued the suggestion from this earlier work that yaw rotation affects primarily the horizontal component. Our results show convincing metric changes in most experiments but also revealed that not all effects were alike.

Figure 4 shows results from an experiment where rotation introduced an obvious change in end point distribution. E-

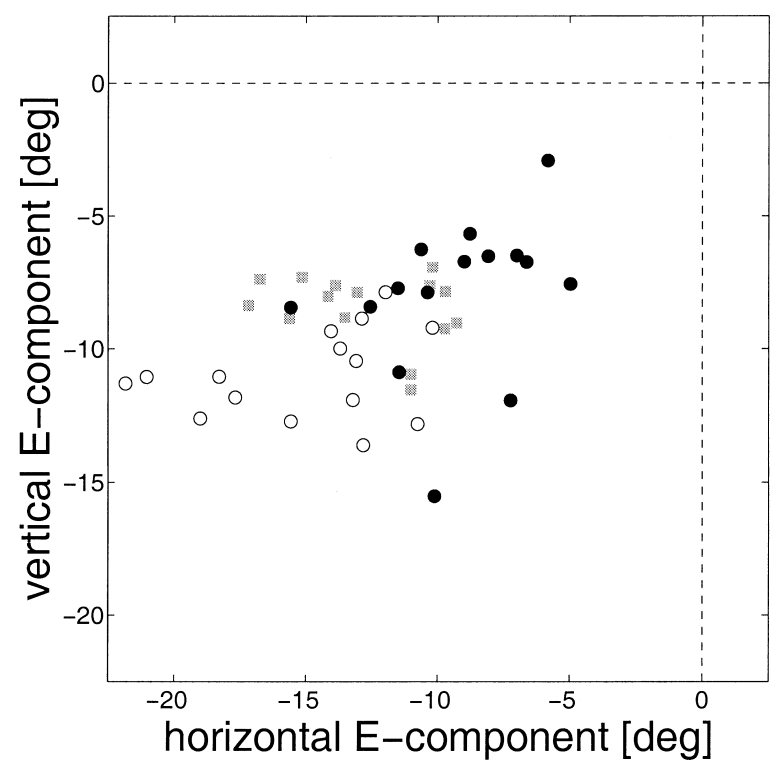

FIG. 4. Example of the effect of yaw rotation on E-saccade metrics. Esaccades elicited during rest ( $⿴$ ) and during leftward $(0)$ and rightward rotation $(\bullet)$ are shown (the 1st 15 saccades recorded in each condition). Note that yaw rotation systematically changed the E-saccade vector. In general, E-saccades elicited during rightward rotation were smaller than the control saccades. E-saccades evoked when the monkey was rotated to the left, into the half field of the E-saccade, were larger. Data from monkey BR. Stimulation duration: $20 \mathrm{~ms}$. 
saccades in the stationary control condition, represented by gray squares, were directed to the left and down. Filled circles indicate E-saccades elicited when the monkey was rotated to the right at chair velocities exceeding $20 \%$; open circles denote saccades elicited during leftward yaw rotation in the same velocity range. Note that during rotation, saccade vectors scattered more widely than the controls. It is clear that saccades elicited during rightward rotation generally had smaller horizontal components than those elicited during leftward rotation, i.e., into the half field of the E-saccade vector. In addition, in this experiment, the vertical component also showed an effect of yaw rotation. So, effectively, yaw rotation into the half field of the E-saccade tended to make it bigger while opposite rotation made it smaller than in the control condition. However, as further analysis will show, just looking at end points may be deceiving since effects of head velocity are superimposed on effects of initial eye position. A statistical analysis was performed to isolate these two effects. First, vestibular stimulation gave rise to nystagmic eye movements that caused some variability in E-saccade starting positions, despite measures to limit this effect (see METHODS). Since it is known that E-saccade vectors may depend on initial eye position (Freedman et al. 1996; Klier et al. 2001; Segraves and Goldberg 1992), it was essential to quantify the impact of this phenomenon on E-saccade metrics. Second, we ascertained to what extent the variability in E-saccade metrics could be related to the direction and magnitude of head velocity. Both factors were investigated separately for horizontal and vertical components.

To quantify how horizontal $\left(\Delta E_{\mathrm{H}}\right)$ and vertical components $\left(\Delta E_{\mathrm{V}}\right)$ of E-saccades in a given experiment were related to initial eye position at saccade onset and to head velocity, we performed a multiple-linear regression

$$
\begin{aligned}
& \Delta E_{\mathrm{H}}=a_{\mathrm{H}} E_{\text {Hini }}+m_{\mathrm{H}} \dot{H}_{\text {yaw }}+b_{\mathrm{H}} \\
& \Delta E_{\mathrm{V}}=a_{\mathrm{v}} H_{\mathrm{Vini}}+m_{\mathrm{v}} \dot{H}_{\text {yaw }}+b_{\mathrm{v}}
\end{aligned}
$$

where $E_{\mathrm{Hini}}$ and $E_{\mathrm{Vini}}$ represent initial horizontal and vertical eye position and $\ddot{H}_{\text {yaw }}$ represents horizontal head velocity. Figure 5 shows the linear regression results for the horizontal (left-hand panels) and vertical (right-hand panels) component of all E-saccades elicited during the same experiment as shown in Fig. 4. For both components there was a reasonable correlation between data and model fit (goodness-of-fit values 0.69 and $0.49, A$ and $B$ ). Model fits were generally better for the horizontal component (mean $R^{2}$ for horizontal $0.47 \pm 0.25$; vertical $0.30 \pm 0.21$, based on all data from the 2 monkeys).

As can be seen from the coefficient values $\left(a_{\mathrm{H}}=-0.45 \pm\right.$ 0.04 and $\left.a_{\mathrm{V}}=-0.55 \pm 0.06\right)$, significant at the $P<0.001$ level ( $t$-test), the experiment in Fig. 5 had a clear E-saccade onset-position effect in both components. To visualize this effect of initial eye position in isolation, the partial-regression plots in Fig. 5, $C$ and $D$, show how variations in E-saccade onset position correlate to changes in component size. The slopes of the regression lines reflect coefficients $a_{\mathrm{H}}$ and $a_{\mathrm{V}}$, respectively. The effects of initial eye position in this experiment were far from negligible, as the range of approximately $10^{\circ}$ in associated component variations indicates. Note that both components showed a similar onset position dependence, with comparable slopes and goodness-of-fit values. The position effect was significant $(t$-test, $P<0.05)$ in the majority of experiments, both for the horizontal (26/37) and the vertical component (33/37).

However, initial eye position accounted only partly for the observed E-saccade scatter. As shown by the partial-regression plots in Fig. 5, $E$ and $F$, there was an additional clear relationship between head velocity and saccade component size. Note that changes in component size increased linearly with head velocity, and that leftward and rightward rotation altered the metrics of the E-saccade in opposite directions. The effect, however, was more pronounced in the horizontal component as expressed by the difference between coefficients $m_{\mathrm{H}}=$ $0.095 \pm 0.006 \mathrm{~s}$ and $m_{\mathrm{V}}=0.025 \pm 0.005 \mathrm{~s}$.

If the two factors in the multiple regression equation (initial eye position and head velocity) are strongly correlated, caution is warranted to avoid erroneous conclusions. Collinearity becomes a possible point of concern for correlations beyond $r=$ 0.80 (Glantz and Slinker 1990). We found that the actual correlations between the two factors remained below this value without a single exception (horizontal: $r=0.40 \pm 0.16$; vertical $r=0.22 \pm 0.15)$. As an additional check, we compared goodness-of-fit values $\left(R^{2}\right)$ for $E q . l$ and a reduced version lacking the head velocity term. The results showed that the head velocity term significantly improved the $R^{2}$ values of the model in 31 of 37 experiments for the horizontal component. This number was considerably smaller for the vertical component (20/37). The partial $R^{2}$ for head velocity ranged from 0.00 to 0.91 (mean: $0.37 \pm 0.28$ ) for the horizontal component. In the vertical component we found a range from 0.00 to 0.37 (mean: $0.09 \pm 0.11$ ). An additional indication that the analysis yielded consistent results is the similarity of the initial eye position dependence in rest and during rotation (correlation coefficient $r=0.90$, slope $0.88 \pm 0.06$ ). The bias (coefficients $b_{\mathrm{H}}$ and $b_{\mathrm{V}}$ ) was also similar during rest and during yaw rotation (correlation coefficient $r=0.99$, slope $0.98 \pm 0.01$ )

We focused the regression analysis on head velocity, but could the relation with head velocity actually represent a hidden relation with head position? Since in a sinewave each head position has two head velocities associated with it, it is unlikely that variations in head position would produce a tight relation with velocity. Indeed, only two experiments that showed a significant relation with head velocity displayed a stronger relation with head position when we did the regression with head position rather than head velocity. Only one experiment without a significant head velocity relation yielded a significant effect of head position. Since head position and head acceleration are perfectly negatively correlated in a sinusoidal profile, this result also argues against a hidden relation with head acceleration in the overwhelming majority of experiments.

In summary, the multiple-regression analysis is an adequate approach to separate and quantify two distinct sources of variability in E-saccades with consistent results. Most sites show a robust relation between the size of the horizontal component and head velocity.

\section{Characteristics of the metric rotation-effect}

In what follows, we found it convenient to visualize the isolated metric effect of head rotation as a vector, termed $\mathrm{M}$-vector. The M-vector was defined as the change in the E-vector in response to a $50 \%$ s rotation into the half field of the 


\section{A horizontal}
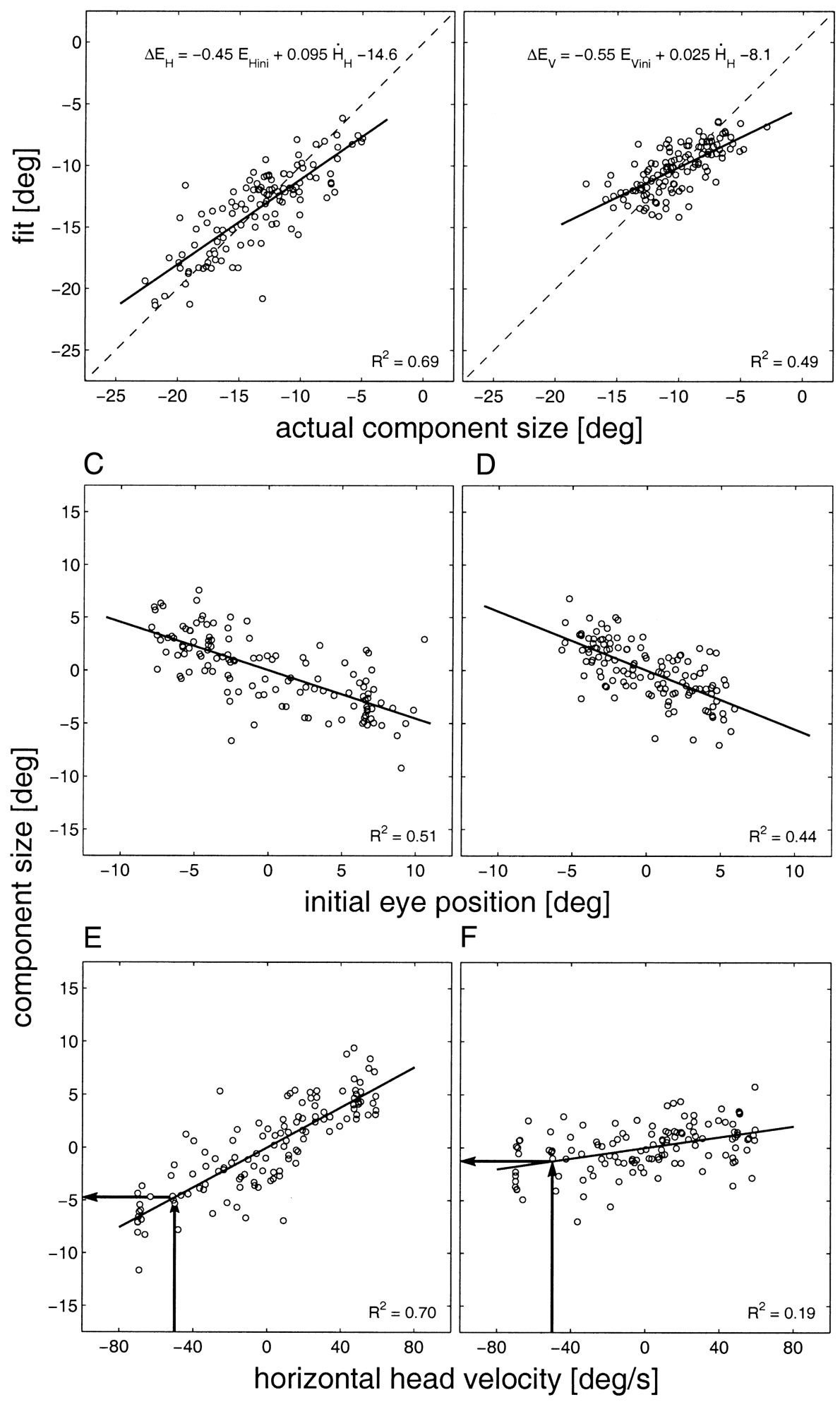

FIG. 5. Combined effect of initial eye position and head velocity on E-saccade metrics. A multiple linear-regression analysis was performed to quantify the relation between E-saccade component size, initial eye position and head velocity (see $E q .1$ ). Together, E-saccade onset position and head velocity can explain a considerable proportion of the variations in both horizontal $(A)$ and vertical $(B)$ component, as can be seen from the goodness-of-fit values ( 0.69 and 0.49 , respectively). A graphic impression of the underlying relationships can be derived from so-called partial regression plots that show how changes in initial eye position $(C$ and $D)$ or head velocity ( $E$ and $F$ ) account for variations in component size, here shown relative to the mean component size. The slopes of the regression lines equal the coefficients found in the multiple regression. Note that an eccentric onset position at E-saccade onset had a clear effect. The effect of head velocity was stronger on the horizontal than on the vertical component. Note that both relationships are linear. Since initial eye position and head velocity have an effect of equal magnitude on E-saccade metrics, end-position plots like Fig. 4, where both effects are expressed superimposed, are of no use to get an impression of the specific effect of head rotation. Arrows in $E$ and $F$ indicate how the slope of the 2 relationships determines M-vector components. Same experiment as in Fig. 4.
E-saccade. As illustrated in Fig. 5, its components were computed by taking $m_{\mathrm{H}} \times-50^{\circ} \%$ for the horizontal (Fig. $5 E$ ) and $m_{\mathrm{V}} \times-50 \%$ s for the vertical component (Fig. $5 F$ ). Note that each experiment yields one M-vector. So, the experiment in Fig. 5 yielded an M-vector with a horizontal component of $-4.75^{\circ}\left(0.095 \mathrm{~s} \times-50^{\circ} / \mathrm{s}\right)$ and a vertical component of $-1.25^{\circ}\left(0.025 \mathrm{~s} \times-50^{\circ} / \mathrm{s}\right)$ that was directed to the left and downward. Since the M-vector was directed into the left half field, just as the E-vector (see Fig. 4), the head-velocity effect was anticompensatory. If the system were to keep the rapid gaze shift constant in the presence of head rotation (see Fig. 2), the M-vector should have been directed away from the E-saccade (rightward in this case). The M-vector was also slightly downward, but less than suggested by the raw data in Fig. 4, 
which still contain the initial eye position effect. Our further analysis will first concentrate on the question to what extent these M-vectors had the appropriate characteristics (sign and amplitude) to maintain E-saccade gaze constancy during head rotation.

In Fig. 6 we show all E-saccade vectors (left-hand column) and the corresponding $\mathrm{M}$-vectors (right-hand column), both for monkey BR (top row) and for monkey GI (bottom row). Since the monkeys were stimulated in different colliculi, their Esaccades were directed into opposite hemifields. M-vectors occupied a much narrower direction range than the E-saccades, mostly close to the horizontal axis.

VIOLATIONS OF GAZE CONSTANCY. Recall that adjusting the E-saccade for the head rotation, in order to keep the gaze shift constant, requires a horizontal $\mathrm{M}$-vector that is directed away from the E-saccade. Such oppositely directed M-vectors were found in 14 of 37 experiments ( $t$-test, $P<0.05$ ), but these vectors were always relatively small. By contrast, in 17 experiments we saw typically very robust anticompensatory effects $(t$-test, $P<0.05)$. In the remaining experiments $(6 / 37)$, the $\mathrm{M}$-vector was not significant.

In Fig. $7 A$ we have pooled the $\mathrm{M}$-vector results from both monkeys, rotating all M-vectors from monkey GI by $180^{\circ}$ as if they were obtained from E-saccades directed into the left hemifield, just as in monkey $B R$ (see Fig. 6, $A$ and $B$ ). The solid line shows the horizontal $\mathrm{M}$-vector component required for gaze-shift constancy, as a function of E-saccade duration. It represents the compensation that would null out a $50 \%$ head rotation occurring during the E-saccade and shows that larger saccades require larger $\mathrm{M}$-vectors because they last longer. The different symbols denote results obtained in the long $(\bullet)$ and short stimulation paradigm (O). The shorter duration paradigm tended to yield smaller shorter-lasting saccades, but, as will become clear, our conclusions apply equally to both experimental conditions.

The total data set in Fig. $7 A$ shows a gradual transition from full compensation (near the solid line) to spectacular violations caused by anticompensatory metric effects (data points below the dashed line). The latter effect predominated as saccade duration increased. Note that full compensation was quite rare and was never observed in saccades above $20^{\circ}$ (durations $>50$ $\mathrm{ms})$. Although a considerable fraction of experiments yielded what we have called compensatory M-vectors (positive horizontal $\mathrm{M}$-vector components), the compensation was mostly incomplete if not negligible. We conclude that, in a clear majority of experiments, vestibular rotation led to violations of spatial accuracy, ranging in a continuum from undercompensation to changes in the wrong direction.

DIRECTIONAL SCATTER. A plausible explanation of the trend toward anticompensatory effects is that the electrical stimulation may have enabled both the saccadic and the quick-phase system. If so, the question arises whether the putative quickphase system contribution had a collicular or a more peripheral origin (see INTRODUCTION). With this issue in mind, we analyzed the directional variability in M-vectors (Fig. 6) from the perspective of two hypotheses, each with different predictions.

The first hypothesis, suggested by Kitama et al. (1992), assumes that the M-vector is aligned with the direction of

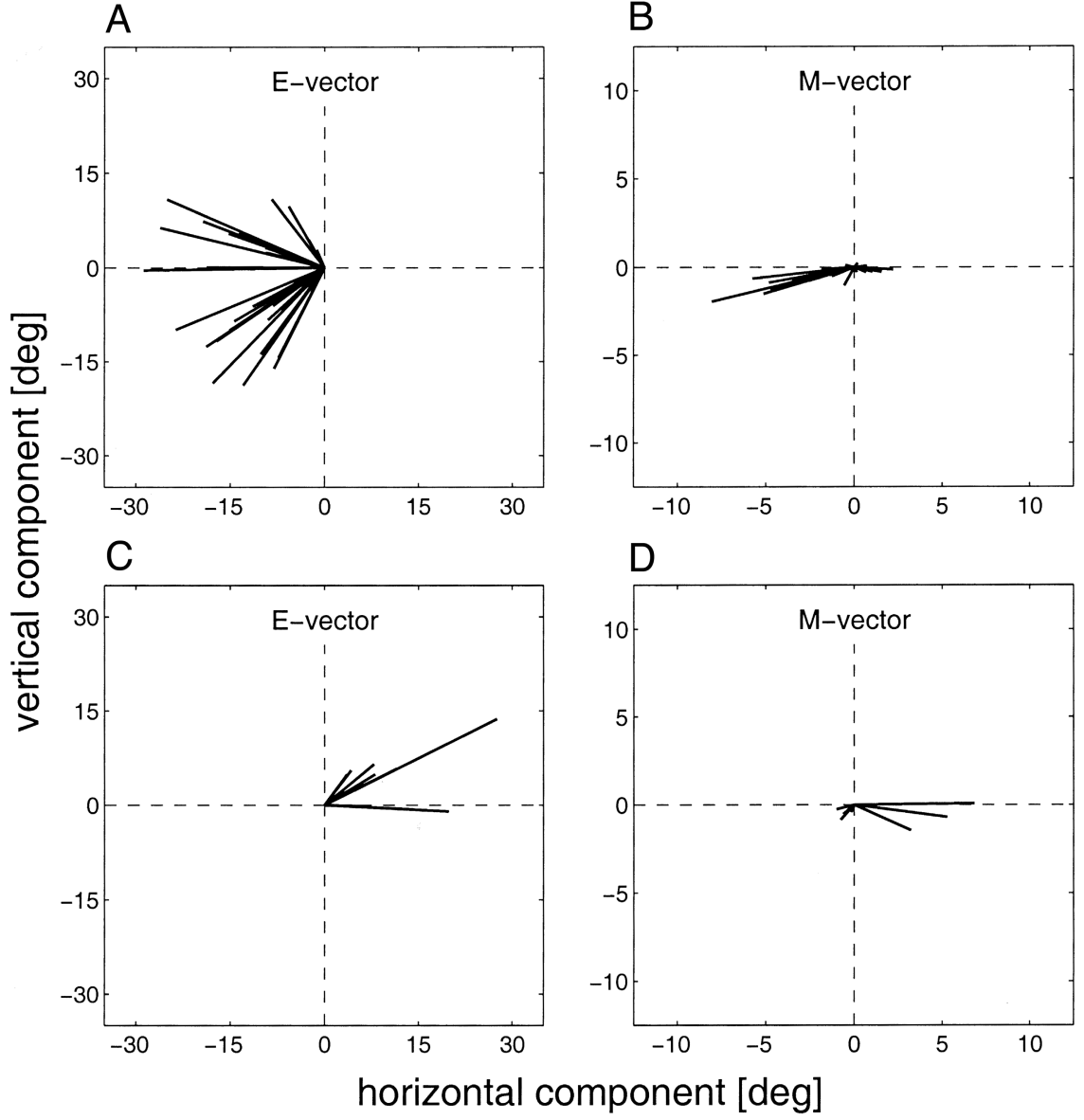

FIG. 6. Overview of E-saccade vectors and M-vectors obtained in the 2 monkeys. Data from all yaw rotation experiments in monkey BR (top panels) and monkey GI (bottom panels). A variety of E-saccades was elicited, with amplitudes up to $\sim 25^{\circ}$, and a wide range of directions $(A$ and $C$ ). This is in clear contrast to the M-vectors, that all lie close to the horizontal axis $(B$ and $D)$, showing that yaw rotation mainly modulated the horizontal component. Note that M-vectors can have amplitudes up to $8.3^{\circ}$, indicating that the vestibular modulation effect was often substantial. Notice also that M-vectors can be directed opposite to the corresponding E-vector. Pooled for both monkeys, the majority of the experiments (BR: 26 of $28 ; G I: 5$ of 9) showed a significant modulation of the horizontal component, whereas a smaller number (BR: 14; GI: 6) exhibited a significant effect on the vertical component $(P<0.05)$. 


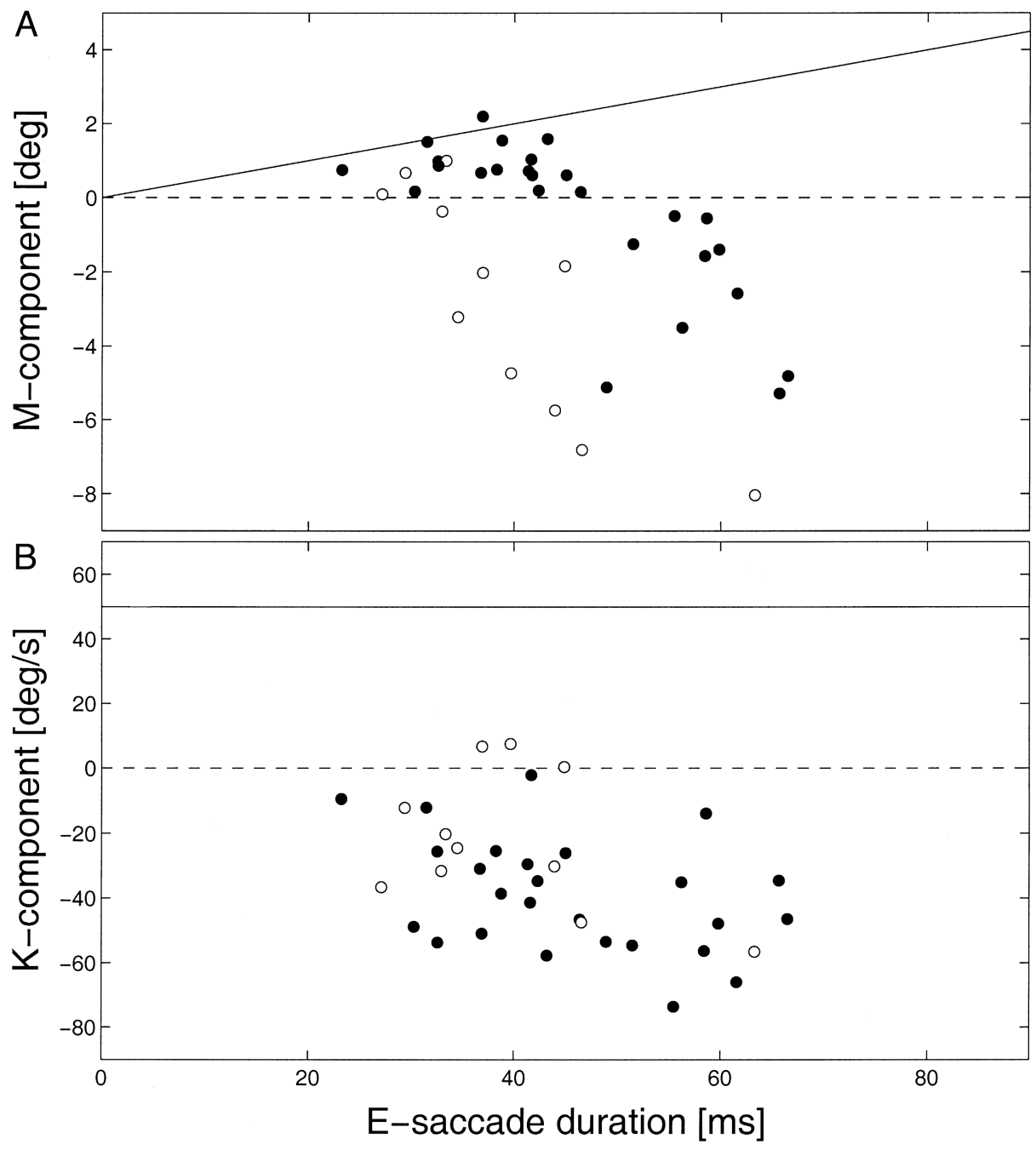

FIG. 7. Lack of spatial constancy during Esaccades. The size of the horizontal M-vector component is shown as a function of E-saccade duration in $A$. The solid line is a prediction of the horizontal M-vector component if a $-50 \%$ sead movement occurring during the E-saccade were completely compensated: e.g., for a 40-ms duration saccade, the horizontal M-vector component would be $0.040 \mathrm{~s} \times 50^{\circ} / \mathrm{s}=2.0^{\circ}$. The points would scatter around the horizontal dashed line if there were no effect of head-rotation at all. Note that full compensation was rare. Using the same format, $B$ shows all horizontal $\mathrm{K}$-vector components from both monkeys. The solid line, corresponding to $K_{\mathrm{h}}=50 \%$, denotes the expected result if horizontal peak velocity in the E-saccade head slowed down during the passive head movement so that gaze peak velocity would remain constant. Actually, virtually all experiments yielded the opposite effect (velocity enhancement). Data points near the dashed line $\left(K_{\mathrm{h}}=\right.$ $0 \%$ s), showing no kinematic effect, are rare. Note that, unlike in $A$, there is no clear relation with E-saccade duration.

rotation and predicts a zero vertical component (see Fig. 8). Adherence to this hypothesis would support the idea that the metric changes reflect signal addition at the component level (see INTRODUCTION). Deviations from horizontal alignment cannot be explained and are seen as noisy variations of unknown
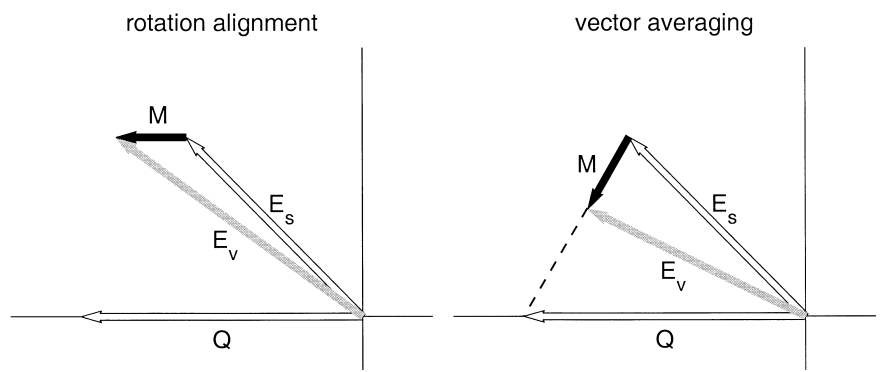

FIG. 8. Schematic illustration of 2 vestibular-saccade interaction hypotheses. The 2 hypotheses predict different effects of yaw rotation on the E-saccade vector. The white vector marked $E_{\mathrm{s}}$ denotes the E-saccade vector during rest, in the absence of vestibular stimulation. The gray vector $E_{\mathrm{v}}$ is the prediction of the E-saccade during a $50 \%$ s leftward rotation that yields leftward quick-phase vector Q. The rotation-alignment idea (left) proposes that only the E-component aligned with the direction of rotation is modulated. So, in the case of yaw rotation, only a horizontal vestibular contribution is added, while the vertical component remains unaffected. The vector-averaging hypothesis (right) assumes that E-saccades during rotation reflect a compromise between the E-saccades during rest and the ongoing quick phases, based on weighted averaging. The M-vector for each hypothesis $\left(M=E_{\mathrm{v}}-E_{\mathrm{s}}\right)$ is also shown. origin. The second scenario to be examined is that the metric effect expressed by the $\mathrm{M}$-vector reflects a compromise between the E-vector and the prevalent quick-phase vector $(\mathrm{Q})$. This idea will be denoted as the averaging hypothesis. If quick phases of nystagmus would obey similar rules of collicular representation as visually guided saccades (see INTRODUCTION), there may have been two loci of SC activity during our experiments: one due to quick phases, the other at the site of the electrode. Since there is evidence that two hills of activity in the SC can cause compromise responses (Edelman and Keller 1998; Glimcher and Sparks 1993; Robinson 1972; Van Opstal and Van Gisbergen 1990), it is interesting to consider what this would predict about M-vectors. If the vestibular metrics effect is based on weighted averaging between Q- and E-vectors, the $\mathrm{M}$-vector should be directed along the line connecting them (see Fig. 8). Since the Q-vector was almost horizontal (see Fig. $3 C$ ), this hypothesis implies that oblique E-vectors should have an $\mathrm{M}$-vector with a vertical component.

In Fig. 9 we have selected results from three experiments to illustrate how the two hypotheses can be evaluated. To do this, we took the actual horizontal component of the M-vector and predicted the corresponding vertical component expected according to each hypothesis. The E-saccade vectors from the three experiments, shown in $A$, were all directed into the left hemifield but ranged in direction from slightly upward to 
A

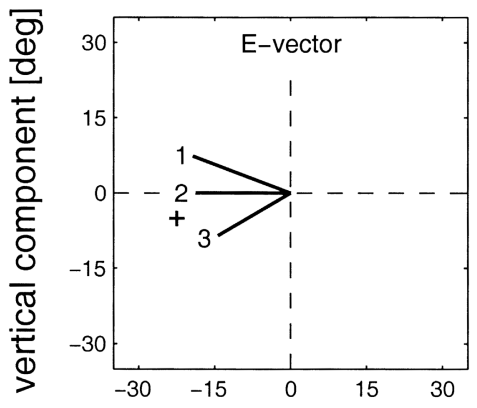

B

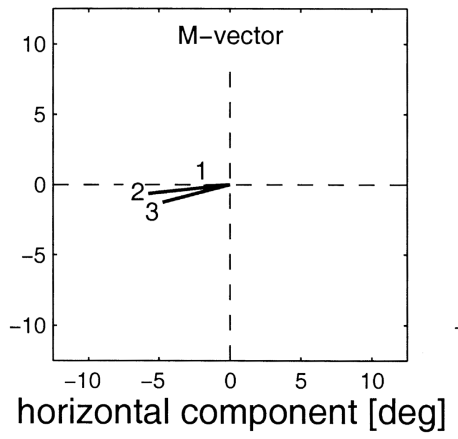

C

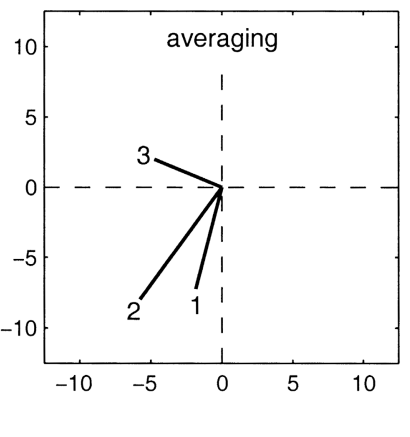

FIG. 9. Illustration of M-vector predictions. A: the E-saccade vectors from 3 experiments in monkey BR. The end point of the average quick-phase vector during head velocities between -45 and $-55 \%$ is marked by a plus sign $(+)$. The effect of vestibular stimulation on E-saccade metrics is expressed in the M-vector (see text for details). The M-vectors obtained in the 3 experiments $(B)$ all point to the left, indicating that rotation into the hemifield of the corresponding E-saccades increased their amplitudes, mainly by modulating the horizontal component. Predictions of the vector-averaging hypothesis are shown in $C$. Note that predictions from the averaging hypothesis do not match the observed M-vectors at all (see text for more details).

somewhat downward. The experiment earlier discussed in Figs. 4 and 5 carries number 3. The actual M-vectors (Fig. 9B) occupied a more narrow range of directions, close to the direction of vestibular rotation (horizontal axis), as the rotation-alignment hypothesis predicts. However, the alignment model is not perfect. The second scheme, which entails vector averaging, requires that the predicted M-vectors are directed along the line connecting the E-saccade vectors and the Qvector. When this direction constraint is combined with the actually observed horizontal M-component to predict the vertical component, these examples show that this scheme fails badly (Fig. 9C).

The next step is to see how the pooled data relates to the two hypotheses outlined above (Fig. 10). When we tried to do this for the averaging idea a problem was encountered. As mentioned above, this hypothesis proposes that E-saccades during rotation reflect a compromise between the putative quick-phase related SC activity, due to vestibular stimulation, and the direct

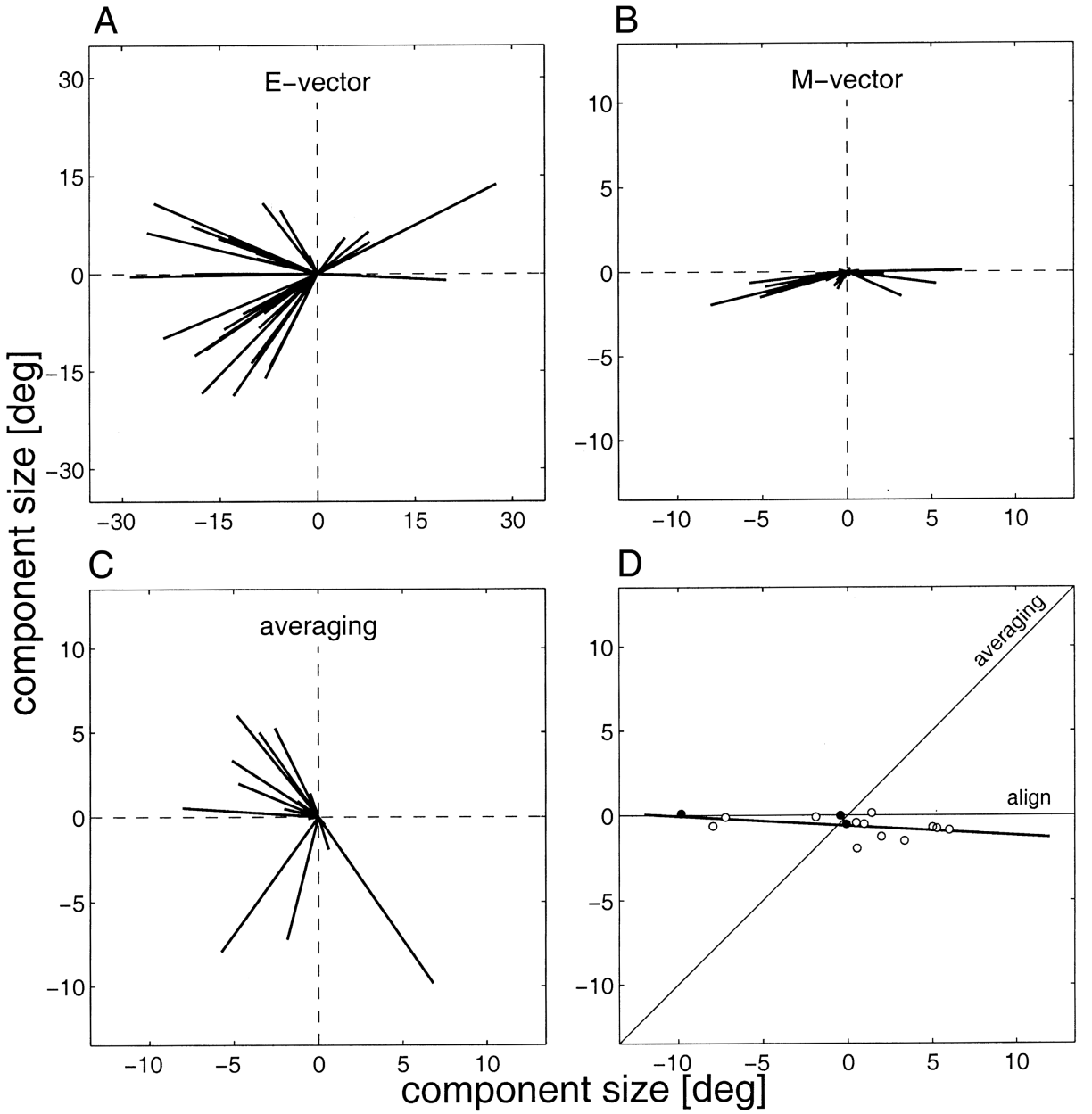

FIG. 10. Summary of yaw-rotation effect on E-saccade metrics. A: all E-saccade vectors, pooled from both monkeys. Saccades were elicited in a wide variety of directions, with different amplitudes. The corresponding M-vectors are shown in $B$. Note that in contrast to the directions of the E-vectors, most M-vectors were close to the horizontal axis, indicating that mainly the horizontal component was modulated by yaw rotation. As explained in the text, we tested 2 vestibular-saccade interaction hypotheses. The $\mathrm{M}$-vector distribution according to the averaging idea is shown in $C$. The predicted $\mathrm{M}$-vector population shows a clear discrepancy with the actual $\mathrm{M}$-vectors $(B)$. To evaluate both schemes quantitatively, $D$ shows the actual vertical M-vector component against the predicted value. If the hypothesis was valid, points would scatter round the unity-slope line (labeled "averaging"). Note, however, that the points actually scatter near the horizontal line, representing no vertical metric changes, as predicted by the rotation-alignment model (labeled "align"). Open circles denote experiments in monkey BR; filled circles represent data from monkey GI. 
TABLE 1. Evaluation of direction predictions of $M$ - and $K$-vectors from two different schemes

\begin{tabular}{lrccccc}
\hline \hline & \multicolumn{3}{c}{ Metrics } & & \multicolumn{2}{c}{ Kinematics } \\
Subject & $n$ & $\begin{array}{c}\text { Rotation } \\
\text { alignment }\end{array}$ & $\begin{array}{c}\text { Vector } \\
\text { averaging }\end{array}$ & & $\begin{array}{c}\text { Rotation } \\
\text { alignment }\end{array}$ & $\begin{array}{c}\text { Vector } \\
\text { alignment }\end{array}$ \\
\hline BR & 28 & 0.67 & 5.26 & & 12.1 & 31.5 \\
GI & 9 & 0.66 & 7.32 & & 18.4 & 34.4 \\
Pooled & 37 & 0.67 & 5.73 & & 13.9 & 32.2 \\
\hline
\end{tabular}

Root-mean-squared errors were calculated for the predictions of the vertical component of the M- and K-vectors (see Figs. $10 D$ and 14C), based on the experimentally determined horizontal components (dimensions: deg and deg/s, respectively). The rotation-alignment scheme performs best in both monkeys, both for the E-saccade metrics and kinematics. Number of experiments is denoted by $n$.

local activation by microstimulation. As a result, E-vectors should end somewhere on the line connecting the E-vector during rest and the Q-vector. Consequently, the horizontal direction of the $\mathrm{M}$-vector required by this model is given by the horizontal components of the E-vector and the Q-vector at a $-50 \%$ rotation. For 22 of 37 of experiments, it was not possible to predict an M-vector according to the vector-averaging hypothesis since the direction of its horizontal component already violated the idea. Further discussion of the averaging predictions will concentrate on the remaining experiments. The rotation-alignment hypothesis was applied to all data.

The vector-averaging hypothesis (Fig. 10C) predicts an Mvector distribution with a much broader range of directions than in the actual M-vectors shown in Fig. 10B. Figure $10 D$ allows a direct comparison between the rotation-alignment and the vector-averaging hypothesis by showing the actual vertical $\mathrm{M}$-vector component against the predicted values. The solid line with unity slope (labeled "averaging") represents the vector-averaging prediction of the vertical M-vector component, based on the actual horizontal component. The horizontal line (labeled "align") shows the prediction from the alignment hypothesis that vertical M-components for yaw rotation will be zero. As can be seen, the actual vertical M-components are generally much smaller than predicted by vector averaging so that this idea has to be rejected. Instead, the data are in much better agreement with the idea that yaw rotation affects only the horizontal component. The slope of the regression line $(0.06 \pm 0.03)$ does not deviate significantly $(t$-test, $P<0.05)$ from the prediction of the alignment hypothesis. To provide a further quantitative comparison, the root-mean-squared residual errors in the prediction of the vertical M-vector components from both schemes are listed in the metrics section of Table 1 . Note that the rotation-alignment hypothesis gave the best description of the data in both monkeys. Its predictions were off by a mere $0.67^{\circ}$, on average.

\section{Specific kinematic changes in E-vectors induced by yaw rotation}

This section will provide evidence to show that, in addition to the metric effect described earlier, vestibular stimulation often also had clear consequences for the kinematic properties of metrically matched E-saccades. Figure 11 shows the relation between component size and component peak velocity for an experiment yielding left-down E-saccades $\left(\Delta E_{\mathrm{H}}=-21.9^{\circ}\right.$, $\left.\Delta E_{\mathrm{V}}=-8.1^{\circ}\right)$. Open circles represent E-saccades during leftward rotation; filled circles denote E-saccades when the monkey was rotated to the right, both at chair velocities exceeding $50 \%$. There was a distinct effect of vestibular stimulation on the kinematic properties of the horizontal component as indicated by the vertical offset in the two clusters. E-saccades with comparable horizontal components exhibited an approximately $100 \%$ s higher horizontal peak velocity during leftward rotations (i.e., into the half field of the E-vector). The vertical component showed hardly any change in peak velocity during leftward rotations (Fig. 11B).

Relying on a multiple-regression analysis, the dependence of horizontal and vertical peak velocity in the E-saccade, $\dot{E}_{\mathrm{H} \max }$ and $\dot{E}_{\mathrm{V} \max }$, on component size $\left(\Delta E_{\mathrm{H}}\right.$ and $\left.\Delta E_{\mathrm{V}}\right)$ and horizontal head velocity $\left(\dot{H}_{\text {yaw }}\right)$ was quantified with

$$
\begin{aligned}
& \dot{E}_{\mathrm{H} \max }=a_{\mathrm{H}} \Delta E_{\mathrm{H}}+k_{\mathrm{H}} \dot{H}_{\text {yaw }}+b_{\mathrm{H}} \\
& \dot{E}_{\mathrm{V} \text { max }}=a_{\mathrm{V}} \Delta E_{\mathrm{V}}+k_{\mathrm{V}} \dot{H}_{\text {yaw }}+b_{\mathrm{V}}
\end{aligned}
$$

For both the horizontal and the vertical component, the regression fits provided a good overall description of the peakvelocity data with goodness-of-fit values of 0.77 for horizontal and 0.80 for vertical (Fig. 12, $A$ and $B$ ). Although it is known that the amplitude peak-velocity relation saturates at large amplitudes, we found that good fits could be obtained using the linear approximation of $E q$. 2, due to the limited amplitude range in a given experiment (mean $R^{2}$ for horizontal $0.69 \pm$

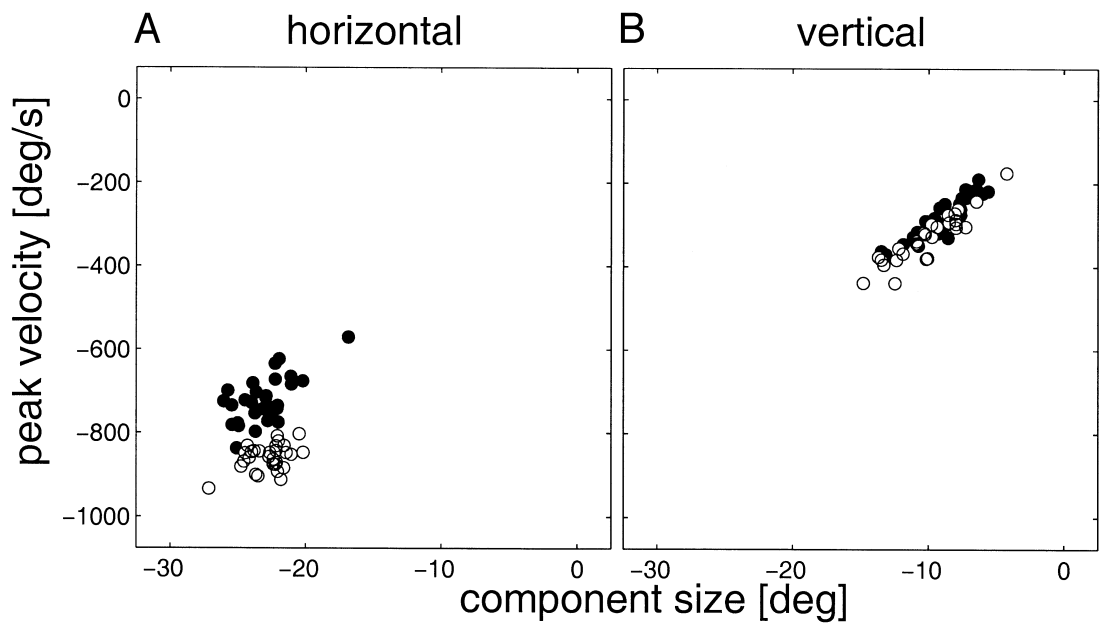

FIG. 11. Illustration of vestibular-stimulation effect on E-saccade kinematics. Component peak velocity is shown as a function of component size for E-saccades elicited during leftward $(O)$ and rightward $(\bullet)$ rotation at rotation velocities exceeding $50^{\circ} \%$ s. E-saccades with equal horizontal components $(A)$ showed an increased peak velocity during leftward rotation (into the half field of the saccade). The kinematic effect in the vertical component $(B)$ was much less apparent. Data from monkey BR. Stimulation duration: $66 \mathrm{~ms}$. 

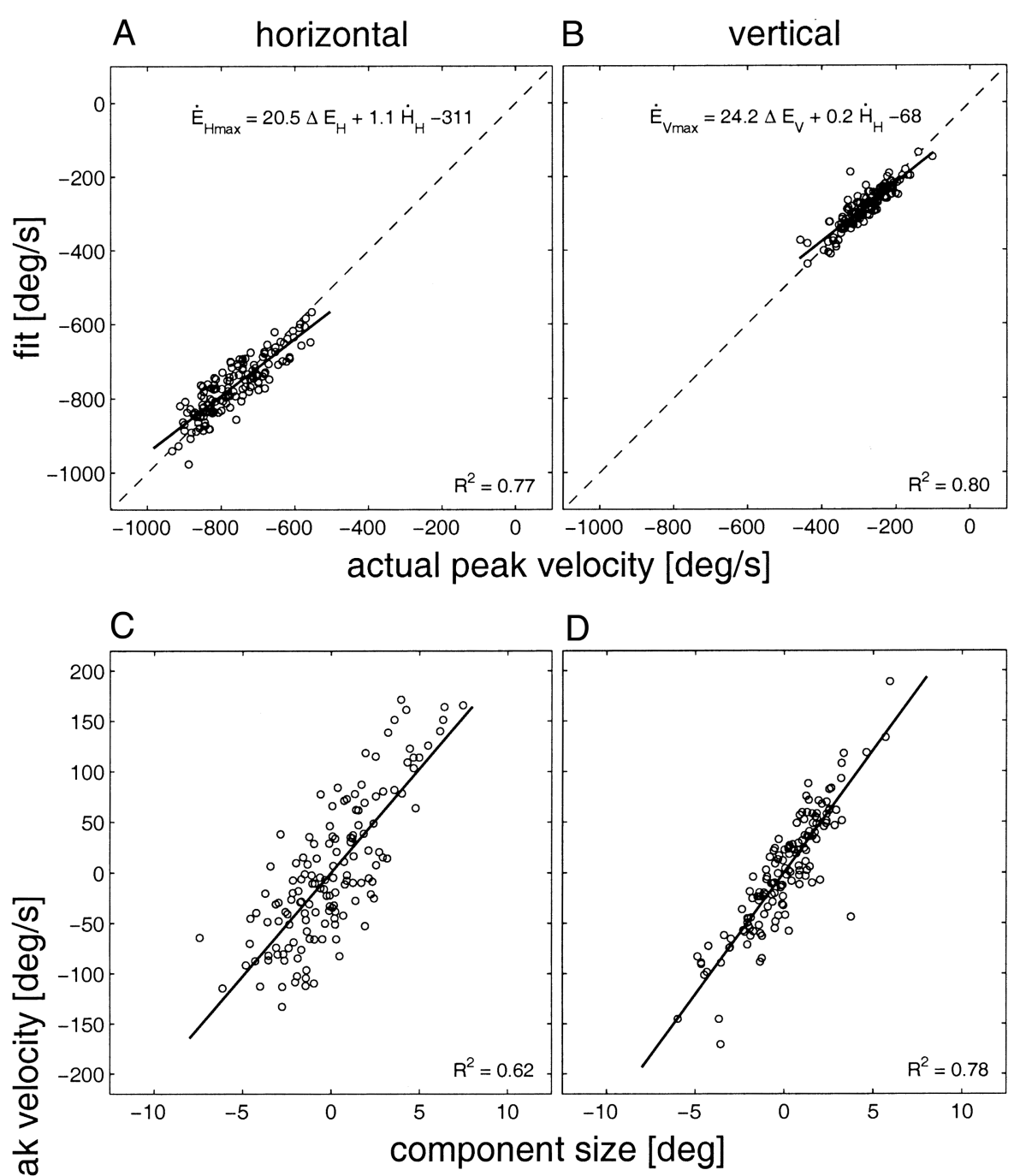

FIG. 12. Statistical separation of component size and head velocity effects on E-saccade kinematics. A multiple linear-regression analysis was performed to quantify the relation between component peak velocity, saccade component size, and head velocity (see $E q .2$ ). This model provides a good overall description of the data for both horizontal and vertical components (goodness-of-fit values 0.77 and 0.80 , respectively). As presumed on the basis of the saccadic main-sequence relation, there was a clear correlation between component size and component peak velocity $(C$ and $D)$. As indicated by the different slopes in $E$ and $F$, the effect of yaw rotation on component peak velocity was more pronounced in the horizontal than the vertical component. Arrows in $E$ and $F$ indicate how the slope of the 2 relationships determines K-vector components. Same experiment as Fig. 11.

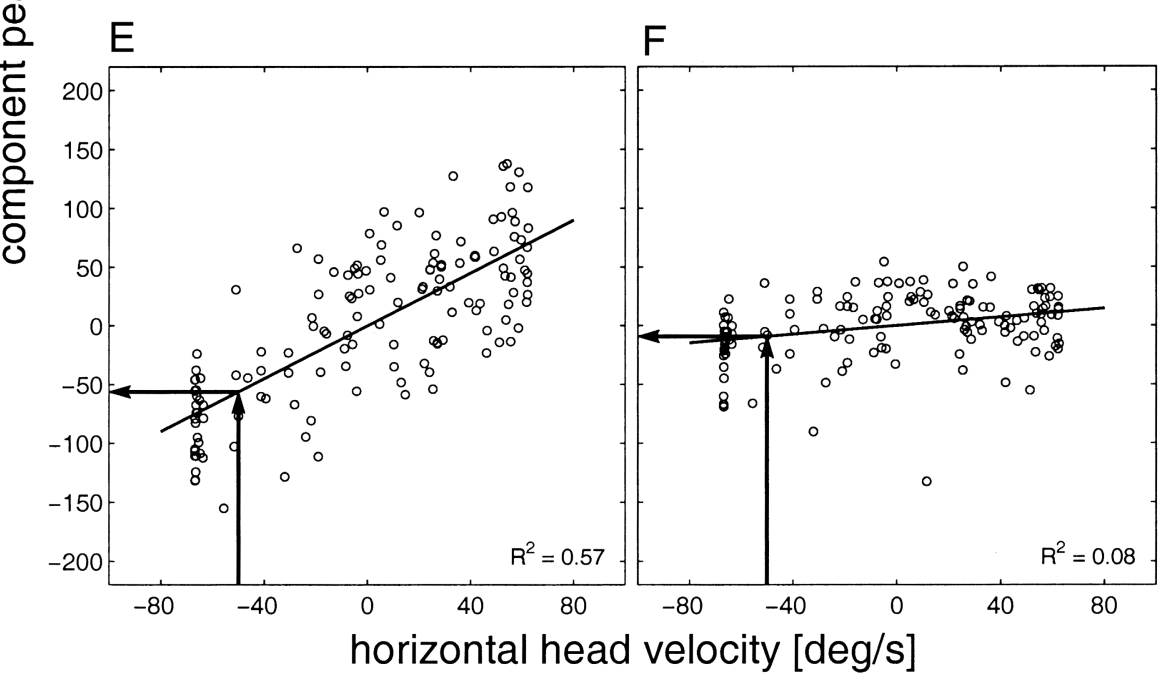

0.17 ; vertical $0.76 \pm 0.10)$. In the experiment of Fig. 12, there was a significant relation between peak velocity and component size as described by coefficients $a_{\mathrm{H}}=20.5 \pm 1.4 / \mathrm{s}$ and $a_{\mathrm{V}}=24.2 \pm 1.1 / \mathrm{s}$ (see partial-regression plots in Fig. 12, $C$ and $D)$. Similar highly significant relations were seen for both components in all experiments (horizontal mean partial $R^{2}$ : $0.58 \pm 0.17$; vertical: $0.74 \pm 0.10$ ). The important result from the multiple-regression analysis is that yaw rotation affected horizontal peak velocity of components of equal size in a linear fashion (Fig. 12E). Note that yaw rotation gave rise to a positive or negative change in peak velocity (up to 10\%), depending on the direction of rotation and head velocity. The vertical component, on the other hand, showed only a minor kinematic effect (Fig. 12F). 
Correlations between the terms of the multiple regression were modest (horizontal: $r=0.49 \pm 0.25$, with 4 experiments exceeding $r=0.80$; vertical $r=0.22 \pm 0.15$ ), indicating that collinearity was generally not a point of concern. The head velocity term contributed a significant increase in $R^{2}$ values for the horizontal component in 30 of 37 experiments, and in 19 experiments for the vertical component. Partial $R^{2}$ values for the head velocity term ranged from 0.00 to 0.75 (mean: $0.27 \pm$ 0.21 ) for the horizontal component and from 0.00 to 0.28 (mean: $0.08 \pm 0.09$ ) for the vertical component.

Figure 13 provides an overview of the effects of vestibular stimulation on E-saccade kinematics in the two monkeys. Using a similar approach as in the metric analysis (see section "Characteristics of the metric rotation-effect"), the change in peak velocity due to a $50 \%$ s rotation into the half field of the saccade, based on the coefficients $k_{\mathrm{H}}$ and $k_{\mathrm{V}}$ as illustrated in Fig. $12, E$ and $F$, was taken as a measure of the kinematic effect (denoted as K-vector). Figure 13, $A$ and $C$, displays the peak-velocity vectors of the E-saccades whose directions show a close resemblance to the E-vectors in Fig. 6, indicating that E-saccades followed a roughly straight path. The corresponding K-vectors (Fig. 13, $B$ and $D$ ) scatter about the horizontal axis, with on average a slight downward component. Kinematic changes were more pronounced in the horizontal component, the horizontal range was approximately three times the vertical range. The typical kinematic effect, seen in almost all (34/37) experiments, was that horizontal peak velocity increased for rotation into the half field containing the E-vector (see also Fig. 12).

To allow comparison with the metric data in Fig. $7 A$, we show the horizontal components of K-vectors from both monkeys as a function of E-saccade duration in Fig. 7B. The solid line denotes the horizontal $\mathrm{K}$-vector component expected in case of addition of a perfect VOR signal, slowing the E-saccade. Instead, the majority of experiments yielded negative horizontal K-components, reflecting increased peak velocities caused by an anticompensatory effect of rotation. K-vectors clearly fail to show the inverse relation with saccade duration seen in M-vectors (Fig. 7A). As a further sign that the metric effect and the kinematic effect are independent, at least to some extent, there were 23 experiments where M-vector and Kvector were directed in opposite hemifields. In other words, irrespective of whether rotation into the half field of the E-saccade made it larger or smaller, the resulting saccade almost invariably showed velocity enhancement.

As Fig. 14, $A$ and $B$, shows, K-vectors clustered more tightly about the horizontal axis than the E-vectors. Nevertheless, since the vertical K-components are not negligible, the question arises how the data should be interpreted. We compared two hypotheses. 1) K-vectors are aligned with the direction of rotation and 2) K-vectors represent a change in vectorial peak velocity. As in the metric analysis, we found that the rotationalignment hypothesis provided the best description of the data (Fig. 14C). The vertical K-vector components predicted on the basis of the vectorial hypothesis (line marked "vector") fail to match the observed data, which are close to the rotationalignment prediction (marked "align"). An analysis of residual errors (listed in Table 1, right-hand side) further substantiated that the rotation-alignment hypothesis was clearly better, in both monkeys.

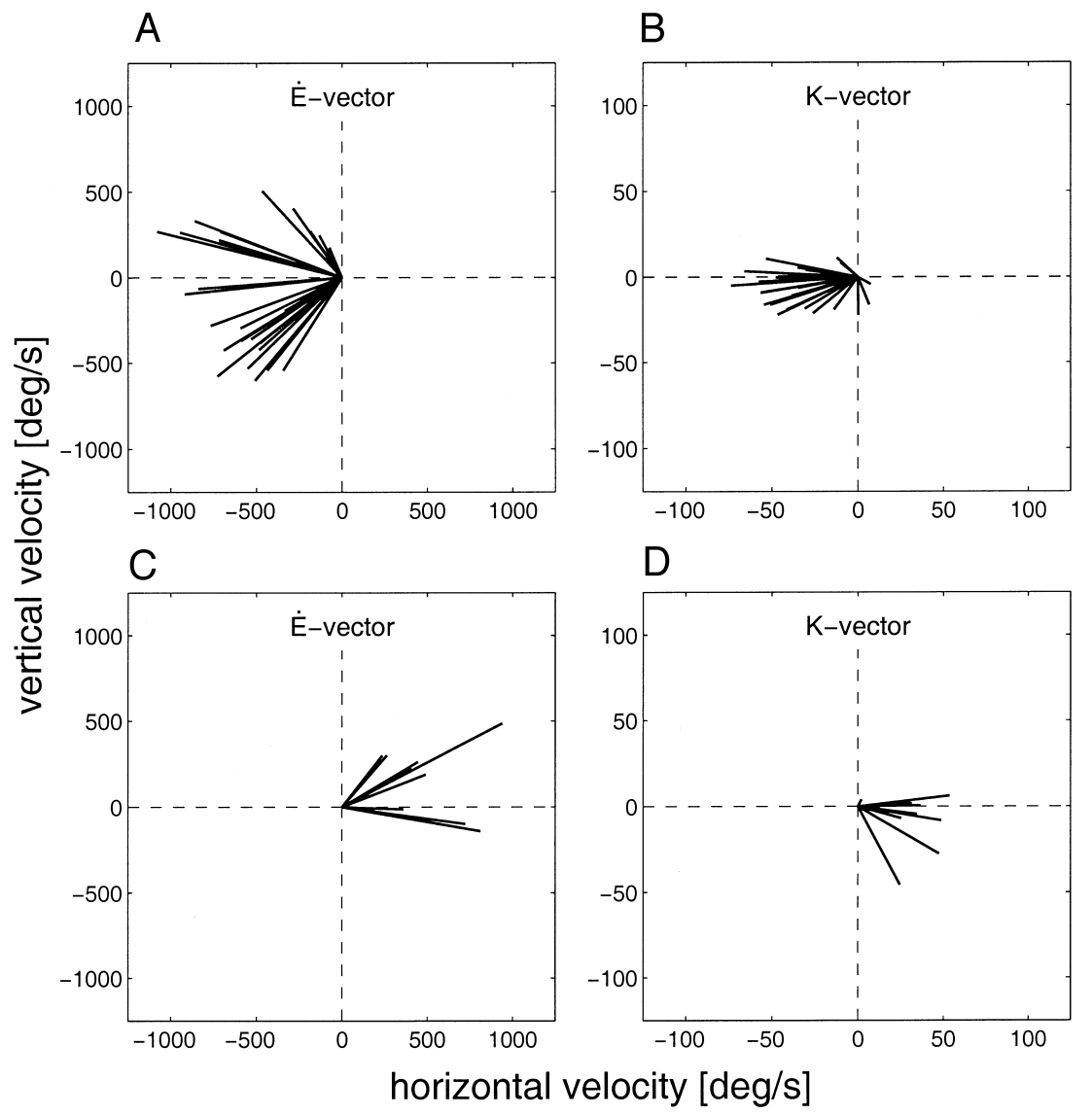

FIG. 13. Overview of E-saccade peak-velocity vectors and corresponding K-vectors for both monkeys. Data from all yaw rotation experiments in monkey BR (top panels) and monkey GI (bottom panels). The directions of E-saccade peak-velocity vectors $(\dot{E})$ closely resemble those of the E-saccade (see Fig. 6, $A$ and $B$ ). In both monkeys, the $\mathrm{K}$-vectors scattered about the horizontal axis $(B$ and $D)$. As indicated by the size of the largest K-vectors, yaw rotation at $50^{\circ} \%$ s could change peak velocity by as much as $70^{\circ} \%$ s. Most experiments (BR: 24 of 28; GI: 7 of 9) showed a significant change in horizontal peak velocity, whereas a smaller number (BR: 16;GI: 3 ) exhibited a significant effect on the vertical component $(P<0.05)$. 

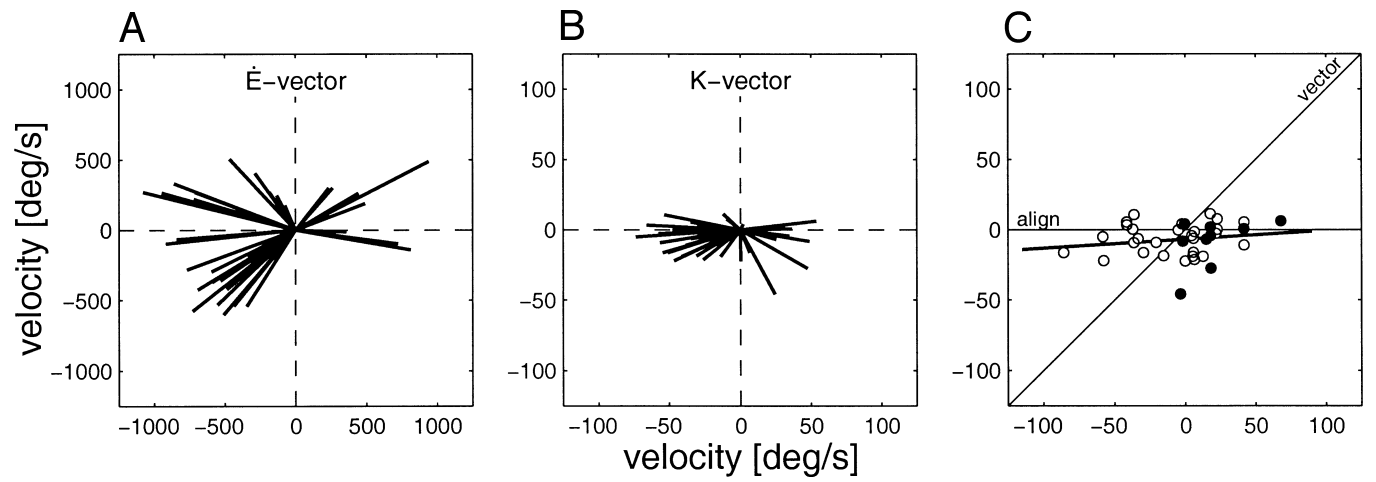

FIG. 14. Effect of yaw rotation on E-saccade kinematics. $A$ : the pooled E-saccade peak-velocity vectors $(\dot{E})$. The corresponding $\mathrm{K}$-vectors are shown in $B$ (see also Fig. 13). The rotation-alignment hypothesis provides the best description for the data. This is illustrated by the relation between the predicted vertical K-vector component and the actual K-vector component $(C)$. Since the vector-averaging hypothesis specifically concerns a metric effect, it was excluded from the kinematic analysis. Open circles denote experiments in monkey BR; filled circles represent data from monkey GI.

\section{Yaw versus pitch rotation}

The yaw-rotation data presented above, strongly suggest that changes in metrics and kinematics of E-saccades predominate in the horizontal component, aligned with rotation direction. To test whether the rotation-alignment hypothesis has a more general validity, we performed separate horizontal and vertical rotation experiments in six different sites and determined Mand $\mathrm{K}$-vectors for each rotation direction. According to the rotation-alignment hypothesis, $\mathrm{M}$ - and $\mathrm{K}$-vectors should be aligned with the horizontal axis during yaw rotation, and be aligned with the vertical axis during pitch rotation.

The top panels of Fig. 15 display the results of the metric analysis. E-saccades had various directions into the left hemifield $(A)$, but most yielded horizontally directed M-vectors during yaw rotation $(B)$. During pitch rotation, E-saccades elicited at the same site showed mainly changes in the vertical component $(C)$. Even more convincing support for the alignment hypothesis was provided by the kinematic analysis (bot-
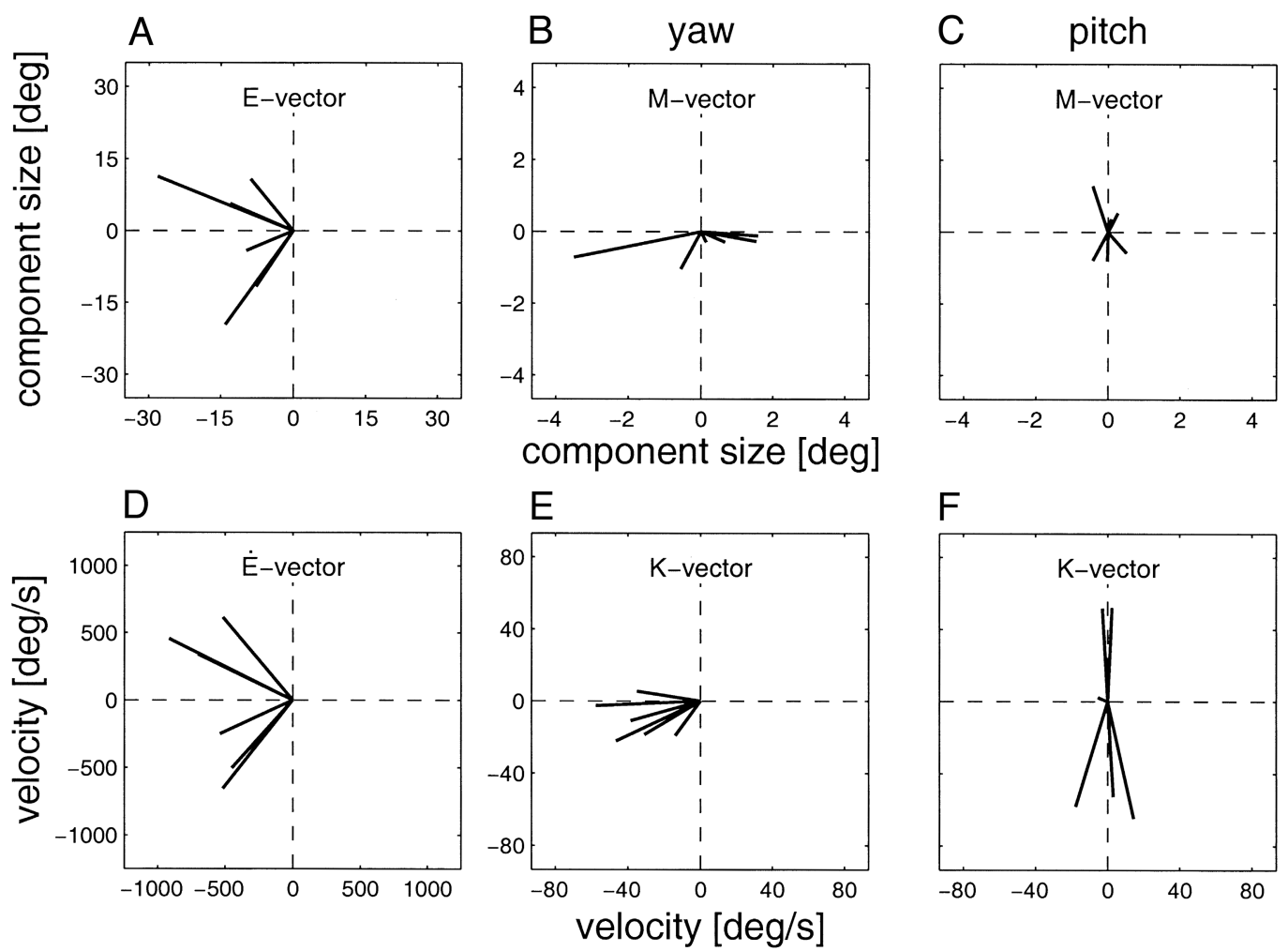

FIG. 15. Comparison of the effects of yaw and pitch rotation on E-saccade metrics and kinematics. E-saccades were elicited at the same stimulation sites $(n=6)$ during yaw and pitch rotation. To further test the rotation-alignment model, we analyzed E-saccade metrics (summarized in the top panels) and kinematics (summarized in the bottom panels). As predicted on the basis of the rotation-alignment model, the M-vectors during yaw and pitch rotation are clearly different. Whereas horizontal rotation results mostly in modulation of the horizontal component, vertical rotation acts mainly on the vertical component. This rotation dependency is also observed in E-saccade kinematics. Only the E-saccade peak-velocity component that is aligned with rotation direction is modulated. The yaw-rotation data from these combined sessions was included in the metric and kinematic analysis of yaw-rotation effects, presented before. Data from monkey $B R$. 
TABLE 2. Evaluation of rotation-alignment and vector-alignment hypotheses on the basis of the combined yaw-pitch experiments

\begin{tabular}{llcccc}
\hline \hline & \multicolumn{2}{c}{ Yaw } & & \multicolumn{2}{c}{ Pitch } \\
\cline { 2 - 3 } \cline { 5 - 6 } & $\begin{array}{c}\text { Rotation } \\
\text { alignment }\end{array}$ & $\begin{array}{c}\text { Vector } \\
\text { alignment }\end{array}$ & & $\begin{array}{c}\text { Rotation } \\
\text { alignment }\end{array}$ & $\begin{array}{c}\text { Vector } \\
\text { alignment }\end{array}$ \\
\hline $\begin{array}{l}\text { Metric } \\
\text { Kinematic }\end{array}$ & $29 \pm 26$ & & $22 \pm 14$ & $59 \pm 26$ \\
\hline
\end{tabular}

Values are means \pm SD. The rotation-alignment idea predicts that the Mand $\mathrm{K}$-vectors are aligned with the direction of rotation (horizontal during yaw rotation and vertical during pitch rotation). The vector-alignment hypothesis entails that $\mathrm{M}$ - and $\mathrm{K}$-vectors are always aligned with $\mathrm{E}$ - and $\dot{\mathrm{E}}$ vectors, respectively, so that they should be the same during yaw and pitch rotation. Numbers denote the angular deviations (in deg) between the model predictions and the actual M- and K-vectors. In line with earlier observations, the rotationalignment model provides the best description of the metric and kinematic data.

tom panels). Note that during yaw rotation all K-vectors pointed to the left, in the same half field as the peak-velocity vectors. By contrast, pitch rotation changed the picture entirely $(F)$. The fact that peak-velocity vectors showed both positive and negative vertical components $(D)$ explains why there were both upward and downward K-vectors during pitch rotation. A summary of a quantitative comparison is listed in Table 2.

\section{I S C U S S I O N}

\section{Overview of main experimental findings}

This investigation into the effect of passive vestibular stimulation on rapid eye movements has shown clear changes in the metrics of E-saccades that were most pronounced in the component aligned with the direction of rotation and were proportional to instantaneous head velocity. The regression analysis yielding this result was designed to rule out that these metric changes were due to changes in the starting position of the E-saccades. Our results confirm the original observation by Kitama et al. (1992) in the cat that vestibular stimulation may give rise to considerable anticompensatory changes (see Fig. 6). However, other experiments yielded the opposite result, compensatory changes in E-saccade metrics, as if the system was attempting to maintain a degree of gaze-shift constancy. The results show that there actually was a gradual transition between these two opposite extremes (see Fig. 7).

A further major finding in the present study is that rotation into the half field containing the E-saccade nearly always made it faster (see Fig. 13). This robust kinematic effect was again essentially limited to the rotation-aligned component, reversed with the direction of rotation and was proportional to head velocity. When the monkey was subsequently rotated in pitch, the metric and the kinematic effect became predominant in the vertical component of E-saccades (see Fig. 15). The kinematic effect is not a trivial epiphenomenon of the metric effect. We took great care to show that it occurred in metrically matched E-saccades. Furthermore, the two effects can even be opposite. In the next section, we try to delimit the neural stage where the convergence of saccadic and vestibular signals may have occurred.

\section{Evidence that the modulation occurs downstream of the SC}

As outlined in the InTRODUCTION, it has been shown that the pathways for saccades to visual, auditory, and tactile targets have already converged at the level of the SC where movement vectors are coded spatially in a motor map. It is not clear whether vestibular quick phases may be controlled by the SC. Neural activity related to quick phases has been demonstrated in the SC (Schiller and Stryker 1972; Wurtz and Goldberg 1972), but its functional significance has always been enigmatic. If the spatial distribution of quick-phase activity conforms to the collicular map, there may have been two loci of collicular activity during our experiments: one due to the electrical stimulation, the other corresponding to the metrics of the quick phases evoked by vestibular stimulation. There is evidence from double-target experiments (Edelman and Keller 1998; Glimcher and Sparks 1993; Van Opstal and Van Gisbergen 1990) and double electrical-stimulation experiments (Robinson 1972) that having two areas of collicular activity may give rise to a phenomenon called saccade averaging, resulting in a compromise movement. If averaging was responsible for the metric modulation effect during vestibular rotation, one would expect the M-vector to be directed toward the site near the horizontal meridian where the vestibular activity locus would be expected (see Fig. 8, right-hand section). As our results have shown, the actual M-vector directions did not square at all with this hypothesis (see Figs. $9 C$ and 10, $C$ and $D$ ). So our study does not provide evidence for a collicular role in the observed interaction effects.

Since we found that the metric and kinematic effects were predominant in the E-saccade component aligned with the direction of rotation (see Figs. 10D and 14C), the most parsimonious explanation is to assume that they occurred at a level where components are specified, rather than at a vectorialcoding stage. If so, the interaction site must have been somewhere downstream of the superior colliculus, in line with earlier models for the generation of quick phases that emphasized the role of brain stem mechanisms (Anastasio 1997; Galiana 1991). All significant kinematic effects indicate involvement of an anticompensatory signal, just as many of the metric findings. In addition, both metric and kinematic changes were proportional to head velocity. To explain these observations, we assume that a head-velocity signal, that carries the appropriate sign, was added to the horizontal component of the E-saccade. More specifically, since BDNs carry such a head velocity signal (see Fig. 1), we suggest that cells of this type (or a neural equivalent) may have been involved. Normally, during vestibular rotation applied in isolation, the head-velocity signal of BDNs will not come to expression since EBNs are shut off by the pause cells. Only if the BDNs carry a burst, their activity will be strong enough to enforce a rapid eye movement (a quick phase). However, the situation becomes different in the conditions of our experiment when the pause cell gate has already been forced open by the collicular E-stimulation. Once the gate is open, we suggest, the added head-velocity signal can augment or decrease the burst cell signal imposed by SC stimulation, depending on the direction of rotation relative to the direction of the saccade. Since the head-velocity signal is only present on horizontal BDNs, only horizontal burst cells would be affected in this fashion, leaving the vertical burst cells unmodified. Such a scheme can nicely explain the kine- 
matic modulation effect typical of virtually all experiments, including the observation that the effect was proportional to head velocity. It should be noticed that the more specific version of our hypothesis can only be preliminary, since BDNs, which have been amply studied in the cat, have only been found in the vertical channel in the monkey, so far (Kaneko and Fukushima 1998).

The involvement of an anticompensatory signal in the kinematic data is obvious (see Fig. 13), but why did this not always lead to anticompensatory metric changes? Recall that compensation for the ongoing head movement was almost never complete and was seen only in smaller E-saccades (see Fig. 7A). Could VOR slow-phase addition have played a role as well, in this amplitude range, by adding a compensatory signal (see Fig. 1)? It is thought that the gain of the VOR is attenuated during large gaze shifts, leaving the VOR active during small saccades (Guitton and Volle 1987; Laurutis and Robinson 1986; Pélisson et al. 1988; Roy and Cullen 1998; Tabak et al. 1996; Tomlinson 1990; Tomlinson and Bahra 1986). By itself, the addition of such a VOR signal may account for the partial compensation seen in small E-saccades (see Fig. 7A). Yet, several phenomena are not well understood at present. First, experiments yielding a compensatory metric effect may still have an anticompensatory kinematic effect. In other words, the size of the horizontal component may decrease, but its peak velocity may still be higher than expected for that amplitude. Second, we found that the sizes of the metric and kinematic effects obtained in the same experiments were uncorrelated and did not share the same relation with saccade duration (see Fig. 7, $A$ and $B$ ). Third, within a given experiment, saccade-bysaccade deviations from the metric regression line (yielding the $\mathrm{M}$-vector) typically were not correlated with deviations with the kinematic regression line (yielding the K-vector). Taken together, these findings indicate that the processes determining E-saccade component amplitude during rotation and those affecting its peak-velocity/amplitude relation are not simply two sides of the same coin. Even when both effects are in the anticompensatory direction, as was often the case, their magnitudes are not rigidly coupled. Settling these questions concerning the precise neural origin of the compensatory and anticompensatory signals awaits further investigation. Our results also raise a quite different issue that we will have to discuss: The occurrence of anticompensatory metric effects appears directly at odds with firmly based notions about the spatial accuracy found in natural gaze shifts.

\section{Spatial accuracy in natural and electrically induced gaze shifts}

An important question is how the oculomotor system can generate accurate gaze shifts, both when the head is still and when it is moving. There is considerable evidence that the gaze control system can produce accurate gaze shifts, even though the head contribution varies from trial to trial (for review, see, e.g., Guitton 1992). It also has become apparent that sudden perturbations of the head movement, as well as passively imposed head movements, are taken into account so that gaze accuracy is maintained (Guitton and Volle 1987; Laurutis and Robinson 1986; Pélisson et al. 1988, 1995; Tomlinson 1990; Tomlinson and Bahra 1986). The problem for the brain, in controlling accurate gaze shifts, is to implement an eye-head strategy that allows all involved subsystems (saccadic system, quick-phase system, VOR, and the head control system) to cooperate.

The question of how this is possible has been subject of extensive investigation and modeling efforts. Bizzi and colleagues (Bizzi et al. 1971; Dichgans et al. 1973; Morasso et al. 1973) suggested that even when the saccadic command for a given target location would always be fixed, independent of whether the head is fixed or free, the variable head contributions would be nulled out by the VOR. Since it appears that the VOR is at least partially suppressed during large gaze shifts (Guitton and Volle 1987; Laurutis and Robinson 1986; Pélisson et al. 1988; Roy and Cullen 1998; Tabak et al. 1996; Tomlinson and Bahra 1986), this so-called linear addition hypothesis fails to explain the data during large gaze saccades. As an alternative explanation of gaze accuracy, several models have suggested that the control signal in the head-free situation is a desired gaze-displacement command (Guitton and Volle 1987; Laurutis and Robinson 1986; Roucoux et al. 1980; Tomlinson and Bahra 1986; for reviews see Becker 1991 and Guitton 1991, 1992). In these schemes, gaze feedback adjusts the saccadic control system for the variable head contributions that may change from trial to trial. However, Freedman (2001) recently proposed a gaze control model that accurately mimics metric and kinematic properties of gaze shifts without relying on gaze feedback.

If the electrical stimulation in the SC evokes a desired gaze shift to a spatially fixed location, the oculomotor system somehow needs to correct the eye contribution for any ongoing head movement to maintain gaze-shift constancy and ensure spatial accuracy. Actually, our results show that in a clear majority of experiments vestibular rotation led to violations of spatial accuracy, ranging in a continuum from undercompensation to changes in the wrong direction (see Fig. 7A). Looking for a plausible source of the anticompensatory vestibular signal, responsible for these violations, we have already proposed that the quick-phase system may have been responsible (see section Evidence that the modulation occurs downstream of the SC). This suggestion is not new: the possibility that the quick-phase system may be active when the head rotates is one of the major features in the models proposed by Barnes (1981) and by Guitton and Volle (1987). In their schemes, the brain stem pulse generator is driven by the saccadic and the quick-phase systems, working in conjunction, but gaze feedback ensures that spatial accuracy is maintained.

Such a contribution from the quick-phase system may have been responsible for "some extra source of innervation" to the pulse generator invoked by Laurutis and Robinson (1986) who investigated the eye contribution during gaze shifts when subjects were passively rotated toward the target. They found that these saccades became faster (reminiscent of our kinematic findings) but nevertheless retained their accuracy (no metric effect): a clear difference with the present results. Our frequent failure to see gaze accuracy is also in stark contrast to several other studies showing that natural gaze saccades to a visual target do maintain spatial accuracy, independent of whether the head movement is normal, perturbed, or passively generated (Guitton and Volle 1987; Pélisson et al. 1988, 1995; Tomlinson 1990; Tomlinson and Bahra 1986). Recently, we have studied saccadic responses to flashed targets in head-fixed human subjects during passive vestibular rotation (Van Beuzekom and 
Van Gisbergen 2002). Refixations to the remembered target often consisted of several saccades and showed clear evidence of compensation for intervening vestibular eye movements.

Furthermore, there is evidence that eye-head coordination mechanisms governing normal gaze shifts to visual targets and those determining electrically induced gaze shifts in the headfree and the head-fixed animal may be different as well. Coimbra et al. (2000) observed in the cat that increasing the moment of inertia of the head led to compensatory changes in the eye saccade when the target was a visual flash, but these adjustments were absent when the gaze shift was elicited by collicular electrical stimulation. Freedman et al. (1996) have emphasized that there are strong similarities in the partitioning of the total gaze shift into eye and head contributions in both paradigms (electrical stimulation against natural behavior) when the head is free to move. For example, the eye contribution in both types of experiment is strongly dependent on initial eye position. However, if the same collicular site is then activated by electrical stimulation while the head is kept fixed, the typical result is that the eye still maintains the same initial eye position dependence without compensating for the lost head contribution. By contrast, when there is a natural target, the eye makes a larger movement to keep the total gaze shift normometric. This discrepancy can be seen as another indication that electrically induced gaze shifts seem to ignore crucial information that is available to the normal system. It is as if gaze shifts caused by electrical stimulation follow rigid default rules for specifying eye and head contributions that fail to take into account that certain conditions may make this default strategy inadequate.

It is still unclear why E-saccades lack the ability of normal saccades to maintain gaze constancy during passive rotation. The hypothesis that the putative gaze feedback system is corrupted during E-stimulation (Coimbra et al. 2000) is interesting but requires rigorous further testing and more refinement. A problem for the model is that an electrically induced gaze disturbance of the natural response to a visual remembered target is properly corrected (Pélisson et al. 1995), indicating that the internal feedback loop can monitor even these artificial movements accurately. Motor strategies based on feed-forward mechanisms, calibrated by training, deserve more attention than they have received so far. As work on saccadic adaptation has shown (Melis and Van Gisbergen 1996), if a natural saccade has been adapted to new circumstances, this change in the control signals does not transfer to comparable E-saccade vectors. Since E-saccades lack several signals accompanying normal saccades, feed-forward contributions recruited by these signals may also be different. For this reason, Freedman's model, which was developed for head-free voluntary gaze shifts (Freedman 2001), cannot be applied to our experimental conditions in a straightforward manner. By providing a tool to corrupt spatial accuracy, the paradigm used in this study may be valuable in further exploration of its underlying mechanisms.

\section{Conclusion}

Our data show that vestibular stimulation modulated E-saccade metrics and kinematics. Since the effect predominated in the E-component aligned with the direction of rotation, we suggest that saccadic and vestibular signals converge down- stream of the colliculus. Addition of an anticompensatory signal, attributed to the quick-phase system, often led to clear violations of spatial accuracy. Why E-saccades deviate from normal saccades in this respect remains to be determined.

The staff of the central animal facility provided excellent care for our monkeys. We thank G. Windau, G. Van Lingen, H. Kleijnen, and T. Van Dreumel for technical support. The authors also acknowledge helpful discussions with C. Kaneko, G. Barnes, and D. Guitton. P. Medendorp gave useful critical comments on an earlier version of the manuscript. Two anonymous referees gave valuable comments that substantially improved the paper.

This work was supported by the Council for Earth and Life Sciences (ALW-NWO).

\section{REFERENCES}

ANASTASIO TJ. A burst-feedback model of fast-phase burst generation during nystagmus. Biol Cybern 76: 139-152, 1997.

BARNES GR. Visual-vestibular interaction in the coordination of voluntary eye and head movements. In: Progress in Oculomotor Research, edited by Fuchs AF and Becker W. Amsterdam: Elsevier, 1981, p. 299-308.

BARNES GR AND PROSSER AJ. Vestibular influence upon head-eye coordination. Ann NY Acad Sci 374: 560-570, 1981.

BECKER W. Saccades. In: Vision and Visual Dysfunction. Eye Movements, edited by Carpenter RHS. London: The Macmillan Press, 1991, vol. 8, p. 95-137.

Bizzi E, Kalil RR, AND Tagliasco V. Eye-head coordination in monkeys: evidence for centrally patterned organization. Science 173: 452-454, 1971.

Bour LJ, VAn Gisbergen JAM, BRuijns J, and OtTes FP. The double magnetic induction method for measuring eye movements: results in monkey and man. IEEE Trans Biomed Eng 31: 419-427, 1984.

Chun KS And Robinson DA. A model of quick phase generation in the vestibuloocular reflex. Biol Cybern 28: 209-221, 1978.

Coimbra AJ, Lefèvre P, Missal M, and Olivier E. Difference between visually and electrically evoked gaze saccades disclosed by altering the head moment of inertia. J Neurophysiol 83: 1103-1107, 2000.

Dichgans J, Bizzi E, Morasso P, and Tagliasco V. Mechanisms underlying recovery of eye-head coordination following bilateral labyrinthectomy in monkeys. Exp Brain Res 18: 548-562, 1973.

Edelman JA AND Keller EL. Dependence on target configuration of express saccade-related activity in the primate superior colliculus. $J$ Neurophysiol 80: 1407-1426, 1998.

FREEDMAN EG. Interactions between eye and head control signals can account for movement kinematics. Biol Cybern 84: 453-462, 2001.

FREEDMAN EG AND SPARKS DL. Activity of cells in the deeper layers of the superior colliculus of the rhesus monkey: evidence for a gaze displacement command. J Neurophysiol 78: 1669-1690, 1997.

Freedman EG, Stanford TR, and Sparks DL. Combined eye-head gaze shifts produced by electrical stimulation of the superior colliculus in rhesus monkeys. J Neurophysiol 76: 927-952, 1996.

Fuchs AF, ReINER D, AND Pong M. Transfer of gain changes from targeting to other types of saccade in the monkey: constraints on possible sites of saccadic gain adaptation. J Neurophysiol 76: 2522-2535, 1996.

GaLiana HL. A nystagmus strategy to linearize the vestibulo-ocular reflex. IEEE Trans Biomed Eng 38: 532-543, 1991.

GANDHI NJ AND KELLER EL. Comparison of saccades perturbed by stimulation of the rostral superior colliculus, the caudal superior colliculus, and the omnipause neuron region. J Neurophysiol 82: 3236-3253, 1999.

Glantz SA and Slinker BK. Primer of Applied Regression and Analysis of Variance. New York: McGraw-Hill, 1990.

GlimCHER PW AND SPARKS DL. Representation of averaging saccades in the superior colliculus of the monkey. Exp Brain Res 95: 429-435, 1993.

GroH JM AND Sparks DL. Saccades to somatosensory targets. II. motor convergence in primate superior colliculus. $J$ Neurophysiol 75: 428-438, 1996.

GuitTon D. Control of saccadic eye and gaze movements by the superior colliculus and basal ganglia. In: Vision and Visual Dysfunction. Eye Movements, edited by Carpenter RHS. London: The Macmillan Press, 1991, vol. 8, p. 244-276.

GuITTON D. Control of eye-head coordination during orienting gaze shifts. Trends Neurosci 15: 174-179, 1992. 
GUITTON D AND Volle M. Gaze control in humans: eye-head coordination during orienting movements to targets within and beyond the oculomotor range. J Neurophysiol 58: 427-459, 1987.

Hepp K, Henn V, ViLis T, AND CoHEN B. Brainstem regions related to saccade generation. In: The Neurobiology of Saccadic Eye Movements, edited by Wurtz RH and Goldberg ME. Amsterdam: Elsevier, 1989, p. 105-212.

Hepp K, Van Opstal AJ, Straumann D, Hess BJ, and Henn V. Monkey superior colliculus represents rapid eye movements in a two-dimensional motor map. J Neurophysiol 69: 965-979, 1993.

JAY MF AND SPARKS DL. Sensorimotor integration in the primate superior colliculus. I. Motor convergence. J Neurophysiol 57: 22-34, 1987.

JudGE SJ, RICHMOND BJ, AND CHU FC. Implantation of magnetic search coils for measurement of eye position: an improved method. Vision Res 20: 535-538, 1980.

KANEKo CR AND FuKUSHIMA K. Discharge characteristics of vestibular saccade neurons in alert monkeys. J Neurophysiol 79: 835-847, 1998.

KeLler EL. Participation of medial pontine reticular formation in eye movement generation in monkey. J Neurophysiol 37: 316-332, 1974.

Keller EL. The brainstem. In: Vision and Visual Dysfunction. Eye Movements, edited by Carpenter RHS. London: The Macmillan Press, 1991, vol. 8, p. $200-223$.

Kitama T, Ohki Y, Shimazu H, Tanaka M, and Yoshida K. Site of interaction between saccade signals and vestibular signals induced by head rotation in the alert cat: functional properties and afferent organization of burster-driving neurons. J Neurophysiol 74: 273-289, 1995.

Kitama T, Shimazu H, Tanaka M, and Yoshida K. Vestibular and visual interaction in generation of rapid eye movements. Ann NY Acad Sci 656: 396-407, 1992.

KLIER EM, WANG H, AND CRAWFORD JD. The superior colliculus encodes gaze commands in retinal coordinates. Nat Neurosci 4: 627-632, 2001.

Kustov AA AND RoBINSON DL. Modified saccades evoked by stimulation of the macaque superior colliculus account for properties of the resettable integrator. J Neurophysiol 73: 1724-1728, 1995.

LAURUTIS VP AND ROBINSON DA. The vestibulo-ocular reflex during human saccadic eye movements. J Physiol (Lond) 373: 209-233, 1986.

MARKHAM CH. How does the brain generate horizontal nystagmus? In: Disorders of the Vestibular System, edited by Baloh RW and Halmagyi GM. Oxford, UK: Oxford Univ. Press, 1996, p. 48-61.

Melis BJ AND VAN Gisbergen JAM. Short-term adaptation of electrically induced saccades in monkey superior colliculus. J Neurophysiol 76: $1744-$ 1758, 1996.

Morasso P, Bizzi E, And Dichgans J. Adjustment of saccade characteristics during head movements. Exp Brain Res 16: 492-500, 1973.

Moschovakis AK, Scudder CA, And Highstein SM. The microscopic anatomy and physiology of the mammalian saccadic system. Prog Neurobiol 50: 133-254, 1996.

NiCHOLS MJ AND SPARKS DL. Nonstationary properties of the saccadic system: new constraints on models of saccadic control. J Neurophysiol 73: 431-435, 1995.

OHKI Y, SHIMAZu H, AND SuZUKI I. Excitatory input to burst neurons from the labyrinth and its mediating pathway in the cat: location and functional characteristics of burster-driving neurons. Exp Brain Res 72: 457-472, 1988.

PÉlisson D, GuitTon D, AND GofFart L. On-line compensation of gaze shifts perturbed by micro-stimulation of the superior colliculus in the cat with unrestrained head. Exp Brain Res 106: 196-204, 1995.

PÉlisson D, PrablanC C, AND URQUizar C. Vestibuloocular reflex inhibition and gaze saccade control characteristics during eye-head orientation in humans. J Neurophysiol 59: 997-1013, 1988.
RoBINSON DA. Eye movements evoked by collicular stimulation in the alert monkey. Vision Res 12: 1795-1808, 1972.

RoBINSON DA. Oculomotor control signals. In: Basic Mechanisms of Ocular Motility and Their Clinical Implications, edited by Bach-y-Rita P and Lennerstrand G. Oxford, UK: Pergamon, 1975, p. 337-374.

Roucoux A, Guitton D, AND Crommelinck M. Stimulation of the superior colliculus in the alert cat. II. Eye and head movements evoked when the head is unrestrained. Exp Brain Res 39: 75-85, 1980.

Roy JE AND Cullen KE. A neural correlate for vestibulo-ocular reflex suppression during voluntary eye-head gaze shifts. Nat Neurosci 1: 404-410, 1998.

SCHILlER PH AND STRYKER M. Single-unit recording and stimulation in superior colliculus of the alert rhesus monkey. J Neurophysiol 35: 915-924, 1972.

Schlag J, Pouget A, Sadeghrour S, and Schlag-Rey M. Interactions between natural and electrically evoked saccades. III. Is the nonstationarity the result of an integrator not instantaneously reset? J Neurophysiol 79: 903-910, 1998.

SCUDDER CA, KANEKo CRS, AND FuCHS AF. The brainstem burst generator for saccadic eye movements. A modern synthesis. Exp Brain Res 142: 439462, 2002.

SEgRAVEs MA AND Goldberg ME. Properties of eye and head movements evoked by electrical stimulation of the monkey superior colliculus. In: The Head-Neck Sensory Motor System, edited by Berthoz A, Vidal PP, and Graf W. New York: Oxford Univ. Press, 1992, p. 292-295.

SPARKS DL. Translation of sensory signals into commands for control of saccadic eye movements: role of primate superior colliculus. Physiol Rev 66: 118-171, 1986.

SPARKS DL AND HaRTwich-Young R. The deep layers of the superior colliculus. In: The Neurobiology of Saccadic Eye Movements, edited by Wurtz RH and Goldberg ME. Amsterdam: Elsevier, 1989, p. 213-255.

Stanford TR, Freedman EG, and Sparks DL. Site and parameters of microstimulation: evidence for independent effects on the properties of saccades evoked from the primate superior colliculus. J Neurophysiol 76: 3360-3381, 1996.

Tabak S, Smeets JB, and Collewijn H. Modulation of the human vestibuloocular reflex during saccades: probing by high-frequency oscillation and torque pulses of the head. J Neurophysiol 76: 3249-3263, 1996.

Tomlinson RD. Combined eye-head gaze shifts in the primate. III. Contributions to the accuracy of gaze saccades. J Neurophysiol 64: 1873-1891, 1990.

TOMLINSON RD AND BAHRA PS. Combined eye-head gaze shifts in the primate. II. Interactions between saccades and the vestibuloocular reflex. J Neurophysiol 56: 1558-1570, 1986.

VAN BEUZEKOM AD AND VAN GISBERGEN JAM. Interaction between visual and vestibular signals for the control of rapid eye movements. J Neurophysiol In press.

VAn Gisbergen JAM, Robinson DA, AND GIELEN S. A quantitative analysis of generation of saccadic eye movements by burst neurons. J Neurophysiol 45 : 417-442, 1981.

VAN OPSTAL AJ AND VAN GISBERGEN JAM. Role of monkey superior colliculus in saccade averaging. Exp Brain Res 79: 143-149, 1990.

WuRTZ RH. Vision for the control of movement. Invest Ophthalmol Vis Sci 37: 2130-2145, 1996.

Wurtz RH AND GOLDBERG ME. Activity of superior colliculus in behaving monkey. III. Cells discharging before eye movements. J Neurophysiol 35: 575-586, 1972.

ZeE DS, Optican LM, CoOK JD, Robinson DA, And Engel WK. Slow saccades in spinocerebellar degeneration. Arch Neurol 33: 243-251, 1976. 